\title{
Diffusion kinetics of lutetium in diopside and the effect of thermal metamorphism on Lu-Hf systematics in clinopyroxene
}

\author{
Elias Bloch $^{1 *}, 2$, James Watkins ${ }^{1}$, Jibamitra Ganguly ${ }^{2}$ \\ ${ }^{1}$ Department of Geological Sciences, University of Oregon, Eugene, OR, 97403, USA. \\ ${ }^{2}$ Department of Geosciences, University of Arizona, Tucson, AZ, 85721, USA. \\ *Correspondence to: elias.bloch@unil.ch \\ Corresponding author present address: \\ Institute of Earth Sciences, Faculty of Geosciences and Environment, University of \\ Lausanne, CH-1015 Lausanne, Switzerland
}

\begin{abstract}
Here we report new experimental data for Lu diffusion in diopside from 1150 $1250{ }^{\circ} \mathrm{C}$ at 1 bar pressure and oxygen fugacity corresponding to the iron-wüstite buffer. The primary experimental dataset defines the following Arrhenius relation for $\mathrm{Lu}$ diffusion parallel to the b-crystallographic axis:

$D_{L u / l b}=1.37( \pm 1.2) \times 10^{-13} \exp \left[208.7( \pm 15.2) \mathrm{kJmol}^{-1} / \mathrm{RT}\right] \mathrm{m}^{2} / \mathrm{s}$
\end{abstract}

We find that Lu diffusion in diopside is significantly faster than that of Hf, which could lead to spurious Lu-Hf ages via decoupling of ${ }^{176} \mathrm{Lu}$ from ${ }^{176} \mathrm{Hf}$ if the system was subjected to protracted periods of high temperature. The extent to which Lu-Hf ages are compromised during initial cooling depends on the peak temperature, grain size, cooling rate and the nature of Lu partitioning between clinopyroxene and its surrounding phase(s); however, in geologically realistic scenarios the effects of diffusion on Lu-Hf ages during initial cooling do not produce Lu-Hf ages that differ from the age corresponding to the closure temperature of Hf by more than a few million years. Lu-Hf 
systematics in clinopyroxene are far more susceptible to diffusion during reheating events that occur after radiogenic ${ }^{176} \mathrm{Hf}$ has already accumulated in the host phase. For a given thermal pulse, the Lu-Hf age varies as a function of grain size, temperature, the timing and duration of reheating and the partitioning of Lu between clinopyroxene and its surrounding phase(s). The data collected and numerical model developed in this study have been used to simulate the effect of thermal metamorphism on the internal Lu-Hf systematics of the eucrites Millbillillie and Piplia Kalan. Although our model results do not offer a comprehensive solution to the apparent pre-solar Lu-Hf ages observed in many ancient meteorites, we argue that the $\sim 4.9$ Ga pyroxene-whole-rock Lu-Hf ages of these specific samples are at least partially, if not primarily, due to diffusive loss of $\mathrm{Lu}$ from the constituent minerals to a partial melt phase during high-temperature metamorphism.

\section{Introduction}

A key assumption in geochronological studies that aim to date igneous crystallization is that the constituent minerals of a given rock behave as closed systems upon crystallization; i.e., that the parent and daughter nuclides are effectively retained in these minerals throughout their existence. However, in many natural systems cooling occurs slowly enough that the daughter product of a given decay system undergoes substantial diffusive re-equilibration between its host mineral and surrounding matrix phases. In the scenario that the daughter product diffuses as fast or faster than the parent nuclide, the resulting age will correspond to the closure temperature $\left(\mathrm{T}_{\mathrm{C}}\right)$ of the daughter product and will post-date the crystallization age of the host mineral (Dodson, 1973; 
Ganguly and Tirone, 1999; Yao and Liang, 2015). Similarly, diffusive exchange undergone during reheating events can lead to partial or total resetting of a given geochronometer. If the daughter product of the decay system of interest is only partially re-equilibrated, then the age recorded by the decay system will likewise be only partially reset. If the daughter product is fully re-equilibrated during the reheating event then the retrieved age will again correspond to the closure temperature of the daughter product during cooling, only in this case the retrieved age will represent cooling from the last thermal pulse during which complete re-equilibration occurred and not cooling from crystallization.

For systems in which the rate of diffusion of the daughter product is indeed greater than or equal to that of the parent isotope, the effects of diffusion on the retrieved age can be quantified provided certain variables are constrained. For example, if the decay system of interest is only partially re-equilibrated during a thermal event, then the extent to which the retrieved age has been reset can be calculated as a function of the temperature-time (T-t) path of the reheating event, the characteristic grain size of the host mineral (i.e. radius or half-width depending on crystal geometry) and the diffusion kinetic parameters of the daughter product (the activation energy $(Q)$ and pre-exponential factor $\left(D_{0}\right)$ specifically) (Ganguly and Tirone, 1999; Bloch and Ganguly, 2014). In cases of complete re-equilibration, closure temperatures can be calculated as functions of diffusivity, peak temperature $\left(\mathrm{T}_{0}\right)$, characteristic grain size and cooling rate (Dodson, 1973; Ganguly and Tirone, 1999; Yao and Liang, 2015).

For geochronological systems in which the daughter product diffuses more slowly than the parent nuclide, the analytical approaches described above are not sufficient to 
quantify the effects of diffusion on the age recorded by the decay system. Diffusive separation of the parent and daughter isotopes, which can occur when a rock is subjected to reheating events or during the initial cooling of a rock, can give rise to spurious apparent ages that can be either too old or too young depending on the magnitude and direction of the net diffusive flux into or out of the phases controlling the slope of the isochron (Kohn, 2009; Kelly et al., 2011; Bloch et al., 2015; Bloch and Ganguly, 2015).

Here we present experimental diffusion data for Lu in diopside, which were collected in conjunction with the diffusion data for $\mathrm{Hf}$ in diopside reported by Bloch and Ganguly (2014). Although clinopyroxene typically has a relatively low Lu/Hf ratio in terrestrial rocks, especially when measured in conjunction with garnet, clinopyroxene often has the highest $\mathrm{Lu} / \mathrm{Hf}$ ratio of the phases used to construct $\mathrm{Lu}-\mathrm{Hf}$ isochrons from extraterrestrial samples (Table 1) and is therefore an important mineral with respect to meteorite geochronology. The data collected in this study have been used in a numerical model to quantify the general extent to which initial cooling and subsequent thermal pulses cause diffusive parent/daughter isotope fractionation of the Lu-Hf decay system in clinopyroxene. In addition, we have applied this model to the thermal metamorphism undergone by the eucrites Millbillillie and Piplia Kalan, in an attempt to explain the apparent pre-solar Lu-Hf ages retrieved from these samples.

The Lu-Hf chronology of the eucrites and several other suites of ancient meteorites has remained somewhat elusive in that this decay system sometimes produces both whole-rock and internal isochron ages that pre-date the accepted formation age of the Solar System by 250-400 million years (Patchett and Tatsumoto et al., 1980; Blichert-Toft et al., 2002; Bizzarro et al., 2003; Thrane et al., 2010; Bast et al., 2012; 
Bizzarro et al., 2012; Lapen et al., 2015; Sanborn et al., 2015), while other isotopic decay systems, and in some cases the Lu-Hf system as well, indicate that these meteorites formed within $\sim 20$ million years after the formation of the Solar System (e.g. Al-Mg: Spivak-Birndorf et al., 2009. Ar-Ar: Bogard and Garrison, 1995. Hf-W: Markowski et al., 2007; Kleine et al., 2012. Mn-Cr: Nyquist et al., 1994; Lugmair and Shukolyukov, 1998; Shukolyukov and Lugmair, 2008. Pb-Pb: Manhes et al., 1984; Lugmair and Galer, 1992; Amelin and Irving, 2007; Amelin, 2008; Amelin et al., 2011b; Brennecka and Wadhwa, 2012. Zircon $U-P b$ : Misawa et al., 2005; Righter et al., 2012; Zhou et al., 2013; Hopkins et al., 2015; Iizuka et al., 2015a. Pu-Xe: Shukolyukov and Begeman, 1996. Rb-Sr: Smoliar, 1993. Sm-Nd: Jacobsen and Wasserburg, 1984; Lugmair and Galer, 1992; Nyquist et al., 1994, Blichert-Toft et al., 2002; Patchett et al., 2004; Boyet et al., 2010; Sanborn et al., 2015. Lu-Hf: Amelin, 2005; Amelin et al., 2011a; Bouvier et al., 2008; 2015; Lapen et al., 2015). The Lu-Hf systematics and thermal histories of the eucrites are not uniform, and we do not propose that preferential diffusive re-distribution of Lu with respect to $\mathrm{Hf}$ is solely responsible for the apparent pre-solar ages of every eucrite Lu-Hf

age; however, we find that this process would have had a significant impact on the Lu-Hf systematics of Millbillillie and Piplia Kalan, and suggest that this mechanism should be evaluated on a case-by-case basis.

\section{Experimental and Analytical Techniques}

Bloch and Ganguly (2014) reported diffusion data for Hf in diopside, and in that study the authors conducted co-diffusion experiments in which diopside crystals were doped 
with both $\mathrm{Lu}$ and $\mathrm{Hf}$. The Lu diffusion data extracted from those samples are reported here. Details of the experimental and analytical procedures can be found in Bloch and Ganguly (2014), but nevertheless a description of these techniques is provided below.

\subsection{Sample preparation and experiments}

The crystals used for the Lu and Hf diffusion experiments were natural gemquality diopsides that were found to be compositionally homogeneous $\left(\mathrm{En}_{47} \mathrm{Wo}_{50} \mathrm{Fs}_{03}\right)$ within the resolution of electron microprobe analyses. Each crystal was cut perpendicular to the a-, b- or c-crystallographic axis, and the cut surfaces were then polished using a combination of mechanical and chemical polishing that has been described in several earlier publications (e.g. Tirone et al., 2005; Sano et al., 2011; Bloch and Ganguly, 2014; Bloch et al., 2015). This polishing regimen produces a mirror polish on the crystal surface and effectively removes the near-surface damaged layer that is likely to develop from the mechanical abrasion of polishing.

After polishing, each sample was sonicated in deionized (DI) $\mathrm{H}_{2} \mathrm{O}$ for 30 minutes and then pre-annealed at or near experimental $T-f\left(O_{2}\right)$ (oxygen fugacity) conditions for a minimum of 24 hours in order to equilibrate the concentration of point defects within the crystal with that of the experimental conditions, and to heal any potential fast-diffusion

paths that may have been generated near the crystal surface during polishing. Subsequent to pre-annealing, the samples were again sonicated in $\mathrm{DI} \mathrm{H}_{2} \mathrm{O}$ for 30 minutes, and then a thin film of the diffusants were deposited onto the polished surfaces by thermally evaporating $\sim 5 \mathrm{mg}$ of $\mathrm{HfO}_{2}$ and $\sim 1 \mathrm{mg}$ of $\mathrm{Lu}_{2} \mathrm{O}_{3}$ under high vacuum $\left(\sim 10^{-8}\right.$ bars $)$. 
Pre-annealing and diffusion-annealing experiments were performed in a vertical gas-mixing tube furnace. The $f\left(\mathrm{O}_{2}\right)$ was regulated by flowing a fixed-ratio $\mathrm{CO}-\mathrm{CO}_{2}$ gas mixture into the furnace tube, and was crosschecked with a zirconia sensor. Without exception, there was excellent agreement between the electromotive force (emf) reading of the sensor and the emf calculated from the imposed $\mathrm{CO} / \mathrm{CO}_{2}$ of the gas. In each experiment, the sample was quickly lowered into the preheated furnace tube, which was then sealed off for the duration of the experimental run. Samples were quenched by moving them to the top of the furnace tube, to promote rapid cooling without oxidation. All experiments in this study were conducted at the iron-wüstite (IW) $f\left(\mathrm{O}_{2}\right)$ buffer.

\subsection{Analytical Protocol}

Subsequent to thermal annealing, the induced Lu and Hf diffusion profiles were measured by depth profiling using a Cameca-IMS 6f secondary-ion mass spectrometer (SIMS) at Arizona State University. Samples were held at $5 \mathrm{kV}$, and were analyzed using an ${ }^{16} \mathrm{O}_{2}{ }^{-}$primary ion beam (PIB) accelerated at $-12.5 \mathrm{kV}$. The PIB was focused to a $\sim 30$ $\mu \mathrm{m}$ spot and then rastered over a $125 \times 125 \mu \mathrm{m}$ area. During analyses, a $-75 \mathrm{~V}$ offset was applied to the sample voltage in order to reduce the number of molecular ions contributing to the total counts of the measured ion. We used an ${ }^{16} \mathrm{O}_{2}^{-}$PIB instead of the more commonly used ${ }^{16} \mathrm{O}^{-} \mathrm{PIB}$ because it significantly reduces the amount of ion beam mixing during SIMS depth profiling (Bloch et al., 2015; Posner et al., 2015; see latter for a detailed description of this phenomenon). No pre-sputtering was conducted in these analyses, but as discussed below the first 10-15 cycles were not used to extract diffusion data due to the presence of a transient zone. Transient zones arise during SIMS depth 
profiling due to the implantation of primary beam ions and subsequent altering of the sample chemistry until a steady state is reached (Williams and Baker, 1981).

A field aperture was inserted into the path of the sputtered ions in order to allow only ions originating from the center of the rastered area to enter the mass spectrometer. After depth profiling was completed, the crater depth was measured with a surface profilometer. The step size within the crystal was then calculated from the measured crater depth, number of measurements and the relative sputtering rates in the crystal and the $\sim 400 \AA$ Alayer of Au deposited onto the crystal surface for dissipation of the charge that develops during depth profiling.

Lu and Hf diffusion coefficients were retrieved by measuring the concentration profiles of ${ }^{175} \mathrm{Lu}$ and ${ }^{180} \mathrm{Hf}$. Experimentally induced diffusion profiles of different isotopes of such heavy elements are not resolvable from one another by any currently available analytical technique. Furthermore, the expected small difference in diffusivity is inconsequential for the purpose of the present study. In addition to ${ }^{175} \mathrm{Lu}$ and ${ }^{180} \mathrm{Hf}$, the concentration profiles of the non-diffusing species ${ }^{26} \mathrm{Mg}$ and ${ }^{30} \mathrm{Si}$ were measured throughout the analyses in order to check the stability of the instrument and infer the locations of the crystal surfaces. It was assumed that the crystal surface is approximately located where the ${ }^{30} \mathrm{Si}$ profile reaches its peak value, as validated by Ganguly et al. (1998). The stability of the instrument during each analysis was checked by monitoring the ${ }^{30} \mathrm{Si}$ and ${ }^{26} \mathrm{Mg}$ count rates during depth profiling. Because both ${ }^{30} \mathrm{Si}$ and ${ }^{26} \mathrm{Mg}$ are nondiffusing species in these experiments, and should thus have flat compositional profiles, no diffusant data were used in the model fits unless the secondary ion intensities of these 
non-diffusing species reached a plateau-like steady state, as illustrated in Fig. 1A. These secondary ion signals were also used to identify the presence of a transient zone.

\subsection{Modeling diffusion profiles}

Most of the experimentally induced diffusion profiles were modeled using the analytical solution to the diffusion equation in the Cartesian coordinate system for diffusion into a semi-infinite medium with homogenous initial composition, $C_{\infty}$, and fixed surface concentration, $C_{s}($ Crank, 1975):

$$
\frac{C_{S} \quad C(x, t)}{C_{S} \quad C}=\operatorname{erf} \frac{x}{2 \sqrt{D t}} \div
$$

where $C(x, t)=$ concentration at point $\mathrm{x}$ and time $\mathrm{t}, \mathrm{x}=$ distance from the crystal surface, $D$ is the diffusion coefficient, and $t$ is the time length of isothermal diffusion. Several Lu profiles from high-temperature experiments were better fit using an instantaneous plane source diffusion model, presumably because there was a greater flux of Lu into the crystal thereby depleting Lu from the crystal surface throughout the experiment. Since all

diffusion in these experiments occurred in the positive spatial direction, the appropriate solution to the diffusion equation is (Crank, 1975):

$$
\frac{C(x, t) C}{C(o, t) \quad C}=\frac{M}{\sqrt{D t}} \exp \left(x^{2} / 4 D t\right)
$$

where $M$ is the initial mass of the diffusing species at the surface ( $\mathrm{x}=0$ ). Equations (1) and (2) represent modifications of the solutions to the diffusion equation given in Crank (1975), where the corresponding solutions assume an initial concentration of zero for the 
diffusing species within the medium. It can be easily verified that these solutions satisfy the diffusion equation and the appropriate initial and boundary conditions.

Because the presence of a transient zone does not permit the use of diffusant data near the crystal surface when SIMS depth profiling is employed (Williams and Baker, 1981), $C_{s}$ and $M$ had to be indirectly inferred in order to calculate $D$. In order to best address this problem, the above equations were linked with an optimization program, MINUIT (James and Roos, 1975), in order to solve for $D$ and $C_{s}$ or $M$ simultaneously such that the solutions give the best least-squares fit to the data; this procedure was developed by Tirone et al. (2005). Reported errors of the diffusion coefficients and the $2 \sigma$ error envelopes of the modeled Arrhenius trends (Fig. 2) were calculated according to standard statistical procedure, as outlined by Tirone et al. (2005) and Sano et al. (2011).

\section{Experimental Results}

Representative Lu and Hf diffusion profiles and model fits to the data from the same diopside sample are shown in Fig. 1 (Hf data are taken from Bloch and Ganguly, 2014). Because of its monoclinic symmetry, diopside is expected to exhibit anisotropic diffusion. In an earlier study it was found that the b-axial direction, which is the only crystallographic axis that coincides with a principal diffusion direction (Nye, 1957), represents the slowest diffusion direction for $\mathrm{Ca}$ and $\mathrm{Mg}$ (Zhang et al., 2010). We carried out experiments parallel to all three crystallographic axes at $1200{ }^{\circ} \mathrm{C}$ to assess the likely extent of diffusion anisotropy. The data (Fig. 1) show no resolvable anisotropy for Hf diffusion, but diffusion of $\mathrm{Lu}$ parallel to the c-axis is considerably faster than that parallel 
to the a- or b-axes. We did not attempt to measure the full diffusion tensor for Lu or Hf in diopside because it is beyond the scope of this study. Statistical treatment of the diffusion data parallel to the b-axis, which constitute the primary data set, in terms of the Arrhenius relation $\left(D=D_{0} \exp [-Q(P) / \mathrm{R} T]\right.$, where $D$ is the diffusion coefficient, $D_{0}$ is the pre-exponential factor and $Q(P)$ is the activation energy of diffusion at pressure $P$ ) yields $D_{0}=1.37( \pm 1.2) \times 10^{-13} \mathrm{~m}^{2} / \mathrm{s}$ and $Q(1 \mathrm{bar})=208.7 \pm 15.2 \mathrm{~kJ} / \mathrm{mol}$ for Lu diffusion parallel to the b-axis in diopside. A summary of the experimental conditions and diffusion data is given in Table 2 .

Time-series experiments were conducted at $1150{ }^{\circ} \mathrm{C}$ in order to provide a permissive criterion for whether the measured diffusion profiles represent volume diffusion or were compromised by other phenomena such as chemical reactions at the crystal surfaces or ion-beam mixing. Lutetium diffusion coefficients plotted against run time at $1150{ }^{\circ} \mathrm{C}$ and the IW $f\left(\mathrm{O}_{2}\right)$ buffer are illustrated in the inset of Fig. 2. All four time-series experiments produced very similar diffusion coefficients regardless of run time (Hf time-series experiments are illustrated in Bloch and Ganguly, 2014), which indicates that the measured concentration profiles were not compromised by interference from non-diffusive processes, and therefore that volume diffusion was indeed the dominant mechanism in producing the observed concentration profiles.

\section{Discussion}

\subsection{Comparison with previous works and anisotropy of Lu diffusion}


There are few studies of rare earth element (REE) diffusion in clinopyroxene. Although this study represents the first measurements of REE diffusion along the b-axis in clinopyroxene at 1 bar pressure, Van Orman et al. (2001) measured the diffusion coefficients of several REEs along the c-axis in diopside. In Fig. 3 we compare their results for $\mathrm{Yb}$ to the $\mathrm{Lu}$ diffusion coefficients measured in this study. The diffusivities of $\mathrm{Lu}$ and $\mathrm{Yb}$ are expected to be very similar due to their identical charge and nearly identical ionic radii (0.977 and $1.019 \AA$ in eight-fold coordination, respectively; Shannon, 1976). Sneeringer et al. (1984) measured Sm diffusion in synthetic diopside at 8,14 and $20 \mathrm{kbar}$, but we have omitted these data from Fig. 3, since these experiments were performed under elevated pressures and on synthetic material.

Lutetium diffusion exhibits considerable anisotropy within the range of temperatures explored in this study and by Van Orman et al. (2001), with diffusion parallel to the c-axis being nearly an order of magnitude faster than that along the a- or baxes at $1200{ }^{\circ} \mathrm{C}$. A noteworthy feature of these data is that, despite exhibiting slower diffusion along the b-axis, the activation energy of $\mathrm{Lu}$ in this direction $(208.7 \pm 15.2$ $\mathrm{kJ} / \mathrm{mol}$ ) is considerably lower than the activation energy of $\mathrm{Yb}$ diffusion along the c-axis $(411 \pm 12 \mathrm{~kJ} / \mathrm{mol})$. According to the compensation law, the logarithm of the preexponential factor $\left(\log D_{0}\right)$ increases linearly with the activation energy of diffusion, especially for diffusion coefficients of different species in a given mineral or of the same species in different minerals (Winchell, 1969). This leads to the intersection of the Arrhenius relations ( $\log D$ vs. $1 / T$ plots) at a temperature known as the compensation temperature $\left(T_{\text {comp }}\right)$, such that at $T<T_{\text {comp }}$ the activation energy increases as the diffusivity decreases and vice versa at $T>T_{\text {comp }}$. 
Taken at face value and accepting the compensation relation, the diffusion data in Fig. 3 would suggest that the temperature regime of the Lu diffusion experiments reported here is above $T_{\text {comp }}$ for diffusion of trivalent REE in diopside (a $T_{\text {comp }}$ of $\sim 1015$ ${ }^{\circ} \mathrm{C}$ is retrieved from extrapolating the Lu diffusion data reported in this study to lower temperatures). However, the diffusion data for several trivalent REEs in diopside reported by Van Orman et al. (2001) do not suggest that $T_{\text {comp }}$ lies below the temperature range of our diffusion experiments. If the compensation relation is valid, then there is a discrepancy between the diffusion data reported in this study and those of Van Orman et al. (2001); however, it is important to bear in mind that the compensation law is an empirical relation and does not have a rigorous theoretical foundation (see Lasaga (1998) for a discussion of the problem). Furthermore, the excellent agreement between our //caxis Lu data and the //c-axis Yb data of Van Orman et al. (2001), coupled with the successful time-series experiments conducted in both studies, indicate that these are indeed robust data.

\subsection{Applications to the Lu-Hf decay system}

For geochronological systems in which the daughter product diffuses more slowly than the parent nuclide, traditional mathematical expressions for quantifying the effect of diffusion on the recorded age, such as closure temperature, are of limited use. The usefulness of the concept of closure temperature is that it provides a way to calculate the temperature of a sample at a specific point in time; the assumption being that the closure temperature of the system of interest corresponds to the age retrieved by that same 
system. However, for systems such as Lu-Hf in clinopyroxene, this assumption is not necessarily valid. Although in a given sample both Lu and Hf will have closure temperatures that can be calculated by traditional formulations as functions of grain size, peak temperature, diffusivity and cooling rate (i.e. Dodson, 1973; Ganguly and Tirone, 1999; Yao and Liang, 2015), there will be a period of time, hereafter referred to as the closure interim, during which $\mathrm{Hf}$ behaves as a closed system but Lu remains open to diffusive exchange with the surrounding matrix phase(s). As a result, the age recorded by this system does not necessarily reflect either the closure temperature of either Lu or Hf. Closure temperatures for $\mathrm{Lu}$ and $\mathrm{Hf}$ in diopside and the resulting closure interims are illustrated in Fig. 4.

As discussed in detail by Bloch and Ganguly (2015), the concept of closure interim is not particularly useful in a quantitative sense because the partitioning of $\mathrm{Lu}$ between the host and adjacent phase(s) has a large effect on the age recorded by the LuHf system. Figure 5 shows the age recorded by the Lu-Hf system in diopside, expressed in terms of deviation from the age corresponding to the closure temperature of $\mathrm{Hf}$, as a function of grain size, peak temperature, cooling rate and Lu partitioning, using the numerical model of Bloch and Ganguly (2015) and a modified version of this model to account for cylindrical geometry. For simulations involving diffusion in a cylinder we have assumed that the cylinder is infinitely long and that all diffusion occurs in the radial direction, as has been assumed in the derivation of analytical closure temperature expressions (Dodson, 1973; Ganguly and Tirone, 1999). Closure temperatures for Lu and Hf calculated using this model are compared with the analytical closure temperature formulation of Ganguly and Tirone (1999) in Fig. 4 in order to provide comparison with a 
known analytical solution. The numerical and analytical closure temperatures vary by no more than $2{ }^{\circ} \mathrm{C}$ in any simulation.

Although Lu diffusion in clinopyroxene is significantly anisotropic, we have not considered anisotropy in the simulations presented in this paper. Closure temperature in anisotropic phases is a considerably more complex problem, and at this time there are no analytical expressions that address this issue. Anisotropic diffusion in monoclinic phases such as diopside is further complicated by the fact that the principal diffusion directions do not coincide with the crystallographic axes, as discussed above. Although this is an interesting and important problem, the main intention of this paper is to address the complexities that arise from geochronological systems in which the parent and daughter nuclides diffuse at significantly different rates.

For simplicity, we have assumed that the distribution of $\mathrm{Lu}$ is homogenous within the host phase. In addition, we have modeled Lu partitioning in terms of $\mathrm{C}_{S} / \mathrm{C}_{0}$, where $\mathrm{C}_{\mathrm{S}}$ is the fixed surface concentration throughout the closure interim and $\mathrm{C}_{0}$ is the initial homogenous Lu concentration within diopside. Although partitioning in natural samples is typically temperature-dependent, this simplification serves to demonstrate the extent to which Lu-Hf ages are effected by this parameter. Model results for garnet that include temperature-dependent partitioning, non-homogenous $\mathrm{Lu}$ and $\mathrm{Hf}$ distributions and moving boundaries can be found in Bloch and Ganguly (2015).

As illustrated in Fig. 5, if partitioning is such that there is no chemical potential gradient between the host and adjacent phases $\left(\mathrm{C}_{S} / \mathrm{C}_{0}=1\right)$, then Lu will remain immobilized throughout the closure interim and the age recorded by the Lu-Hf system will correspond to the closure temperature of Hf $\left(\right.$ age $@ \mathrm{~T}_{\mathrm{C}}(\mathrm{Hf})-\mathrm{Lu}-\mathrm{Hf}$ age $\left.=0\right)$. Strictly 
speaking, there will be a small amount of Lu uptake even if $\mathrm{C}_{\mathrm{S}} / \mathrm{C}_{0}=1$, due to decay of ${ }^{176} \mathrm{Lu}$, but the effect of this $\mathrm{Lu}$ flux is negligible. Inputting values of $\mathrm{C}_{\mathrm{S}} / \mathrm{C}_{0}$ that deviate substantially from unity can produce spurious Lu-Hf ages, but the variation of these ages is not significant unless $\mathrm{C}_{S} / \mathrm{C}_{0} \gg>1$ or $\mathrm{C}_{S} / \mathrm{C}_{0}<<1$. This is partly due to the fact that conditions that are increasingly conducive to a large flux of Lu (higher peak temperature, slower cooling rate, smaller grain size) are increasingly conducive to re-equilibration of Hf isotopes as well, which will lead to a competing effect in terms of the age recorded by the Lu-Hf system. If $\mathrm{Hf}$ isotopes are fully re-equilibrated, then the Lu-Hf age will once again correspond to the closure temperature of $\mathrm{Hf}$.

Another reason that the ages produced by the model results illustrated in Fig. 5 show relatively minor age deviations is that in these simulations it was assumed that all diffusion of Lu and Hf occurs during the initial cooling of clinopyroxene; "initial cooling" here refers to either cooling from crystallization or cooling from the last reheating event during which Hf was fully re-equilibrated between clinopyroxene and its adjacent phases. In the instance of a reheating event that significantly postdates the initial cooling, diffusive loss or gain of Lu from clinopyroxene would make a more significant impact on the recorded Lu-Hf age due to the fact that the ${ }^{176} \mathrm{Hf} /{ }^{177} \mathrm{Hf}$ values of clinopyroxene and the surrounding phases will have diverged over time. For example, Fig. 6 illustrates the effect of two end-member scenarios that would lead to the minimum and maximum effects of diffusion on the Lu-Hf system. The minimum amount of disruption would occur in the hypothetical scenario that all diffusion occurred instantaneously, and coincided with the beginning of radiogenic ${ }^{176} \mathrm{Hf}$ accumulation in clinopyroxene. Diffusion under these conditions would lead to a horizontal displacement 
of the bulk ${ }^{176} \mathrm{Lu} /{ }^{177} \mathrm{Hf}$ ratio in clinopyroxene along the initial ${ }^{176} \mathrm{Hf} /{ }^{177} \mathrm{Hf}$ values, and would not make any impact on the recorded Lu-Hf age (Fig. 6A). Similarly, the maximum disruption would occur if all diffusion is again instantaneous but instead occurs at $0 \mathrm{Ma}$ (Fig. 6C). Although neither of these end-member scenarios are realistic, they serve to illustrate that the timing of diffusion is an important variable and that its effect has both and upper and lower bound.

The extent to which disruption of the Lu-Hf systematics in clinopyroxene depends on the timing and duration of reheating, imposing different values of $\mathrm{C}_{\mathrm{S}} / \mathrm{C}_{0}$, is illustrated in Fig. 7. In order to isolate these variables, the simulations shown in Fig. 7 imposed that no diffusion occurred during the initial cooling of clinopyroxene. As such, we have expressed the model results in terms of (True age - Lu-Hf age), where the true age refers to the time at which clinopyroxene formed. The effect of the timing of reheating is illustrated in Figs. 7A-B and 7C-D for spherical and cylindrical geometries, respectively. As expected, the deviation of the Lu-Hf age from the true age increases with lateroccurring reheating, and with the extent to which $\mathrm{C}_{\mathrm{S}} / \mathrm{C}_{0}$ diverges from unity. All simulations in Figs. 7A-D imposed isothermal reheating events with a duration of 5 Myr.

Figures 7E and 7F illustrate how the Lu-Hf age recorded in clinopyroxene varies as a function of the duration of reheating. For simulations which impose $\mathrm{C}_{\mathrm{S}} / \mathrm{C}_{0}<1$, increased duration of reheating initially leads to increasingly older Lu-Hf ages due to increased Lu loss. At some critical duration Hf isotopes will begin to be effectively reequilibrated, and at increasingly longer durations beyond this point progressively younger Lu-Hf ages will be recorded. In all of the simulations shown in Fig. $7, \mathrm{C}_{S} / \mathrm{C}_{0}$ values $>1$ have a larger effect on the Lu-Hf age than simulations imposing $\mathrm{C}_{\mathrm{S}} / \mathrm{C}_{0}$ values $<1$. This is 
due to the fact that the effect of Lu uptake is enhanced by the re-equilibration of Hf isotopes. Regardless of the $\mathrm{C}_{\mathrm{S}} / \mathrm{C}_{0}$ value, if $\mathrm{Hf}$ isotopes are fully re-equilibrated then the Lu-Hf age will reflect the time at which the reheating event ceased.

\subsection{Application to the eucrites Millbillillie and Piplia Kalan}

In order to demonstrate the effect of thermal metamorphism on measured Lu-Hf systematics from natural samples, we have applied the numerical model developed in this study to the whole-rock pyroxene Lu-Hf ages of the eucrites Millbillillie and Piplia Kalan reported by Bast et al. (2012). As mentioned in the introduction, the Lu-Hf chronology of many eucrites and several other suites of ancient meteorites produces both whole-rock and internal isochron ages that pre-date the accepted formation age of the Solar System by $\sim 250-400$ million years. Although early attempts to resolve this issue focused on uncertainty in the decay constant of ${ }^{176} \mathrm{Lu}$, for which different values were retrieved depending on whether it was calculated based on analysis of terrestrial material (Scherer et al., 2001; Söderlund et al., 2004) or meteorites (Patchett and Tatsumoto, 1980; Blichert-Toft et al., 2002; Bizzarro et al., 2003), this issue has since been addressed (Amelin, 2005; Söderlund et al., 2004; Bouvier et al., 2008) and generally is no longer considered a plausible explanation for the apparent excess ${ }^{176} \mathrm{Hf}$ in these samples.

Alternatively, it has been argued that the unduly steep slopes of these Lu-Hf isochrons could reflect accelerated decay of ${ }^{176} \mathrm{Lu}$ during the first few million years after Solar System formation. Such accelerated decay would require a large-scale irradiation event during or shortly after Solar System formation (Albarède et al., 2006; Thrane et al., 
2010) to convert a substantial fraction of ${ }^{176} \mathrm{Lu}$ (half-life $\approx 37$ billion years) to the fasterdecaying nuclear isomer ${ }^{176 \mathrm{~m}} \mathrm{Lu}$ (half-life $\approx 3.65$ hours), both of which decay to ${ }^{176} \mathrm{Hf}$. This mechanism was first proposed by Albarède et al. (2006), who presented a $\gamma$-ray irradiation model to account for the excess ${ }^{176} \mathrm{Hf}$ observed in whole-rock chondrite and eucrite Lu-Hf isochrons. More recently, several studies have produced internal Lu-Hf isochrons from eucrites and angrites that also pre-date Solar System formation (Thrane et al., 2010; Bizzarro et al., 2012; Bast et al., 2012; Lapen et al., 2015). Because the angrite Sahara (SAH) 99555 has a reconstructed burial depth greater than that which a $\gamma$-ray irradiation event is expected to penetrate, Thrane et al. (2010) proposed that the observed excess ${ }^{176} \mathrm{Hf}$ is due to a spray of cosmic rays following a supernova(e) event, which would also result in the conversion of ${ }^{176} \mathrm{Lu}$ to ${ }^{176 \mathrm{~m}} \mathrm{Lu}$.

While physically plausible, the accelerated decay hypothesis has been challenged in several geochemical studies: As shown by Amelin (2015) and lizuka et al. (2015a), the Lu-Hf systematics of at least some zircons and phosphates from these meteorites are consistent with the decay of non-isomeric ${ }^{176} \mathrm{Lu}$ since the formation of the Solar System. Similarly, Amelin et al. (2011) produced a whole-rock Lu-Hf isochron from angrite samples that did not show evidence for excess ${ }^{176} \mathrm{Hf}$. In addition, isotopes with large neutron-capture cross sections may show deficits in ancient meteorites if an intense spray of cosmic rays did occur (Sanborn et al., 2015). For example, Sprung et al. (2010) found coupled variations of ${ }^{180} \mathrm{Hf} /{ }^{177} \mathrm{Hf}$ and ${ }^{149} \mathrm{Sm} /{ }^{152} \mathrm{Sm}$ in lunar samples that were exposed to cosmic rays. It was also shown by Sprung et al. (2013) that exposure to cosmic rays raises the ${ }^{179} \mathrm{Hf} /{ }^{177} \mathrm{Hf}$ ratio within samples. Sanborn et al. (2015) found only modest ${ }^{149} \mathrm{Sm} /{ }^{152} \mathrm{Sm}$ deficits in the angrites D'Orbigny, and also pointed out that the ${ }^{178} \mathrm{Hf} /{ }^{180} \mathrm{Hf}$, 
${ }^{179} \mathrm{Hf} /{ }^{177} \mathrm{Hf}$ and ${ }^{180} \mathrm{Hf} /{ }^{177} \mathrm{Hf}$ ratios reported for SAH 99555 by Bizzarro et al. (2012) are the same within errors as the terrestrial Hf isotopic composition. Lastly, Wimpenny et al. (2015) found no difference in the ${ }^{176} \mathrm{Lu} /{ }^{175} \mathrm{Lu}$ ratio of achondrites (cumulate and basaltic eucrites and angrites) with respect to terrestrial materials, which would be expected as a consequence of accelerated ${ }^{176} \mathrm{Lu}$ decay.

As described in Table 1, the internal Lu-Hf systematics of both eucrites and angrites vary considerably between samples. In many cases the internal Lu-Hf isochrons yield ages that coincide with the accepted age of solar system formation; in others there is evidence of excess ${ }^{176} \mathrm{Hf}$ from statistically significant isochrons, and several samples produce Lu-Hf ages that are relatively young. Although the process(es) responsible for the Lu-Hf systematics of these samples is the subject of ongoing debate (Martin et al., 2013; Bouvier et al., 2015; Bast et al., 2016), these meteorites show variable degrees of disruption regarding their Lu-Hf isotope systematics. Furthermore, based on these data it seems reasonable to suspect that there may have been multiple mechanisms at work (e.g. accelerated decay \pm reheating \pm some other unidentified process), not all of which were undergone by every sample. The Lu-Hf systematics of Millbillillie and Piplia Kalan differ from the other samples described in Table 1 in that the high ${ }^{176} \mathrm{Lu} /{ }^{177} \mathrm{Hf}$ phases (whole-rock pyroxene) yield pre-solar ages, while low ${ }^{176} \mathrm{Lu} /{ }^{177} \mathrm{Hf}$ phases (ilmeniteplagioclase-composite-whole rock) produce much younger ages of $\sim 3.7 \mathrm{Ga}$. The fact that excess ${ }^{176} \mathrm{Hf}$ is not correlated with $\mathrm{Lu} / \mathrm{Hf}$ ratio in these samples indicates that either accelerated decay did not affect Millbillillie and Piplia Kalan, or that whatever amount of accelerated decay occurred was overprinted by a different process. Terrestrial weathering 
of Millbillillie and Piplia Kalan is not expected to be a significant contributing factor because they were both witnessed falls (Bast et al., 2012).

The Lu-Hf systematics of Millbillillie and Piplia Kalan would have been particularly susceptible to reheating events, due to their small average pyroxene radii of $\sim 0.4$ and 0.5 mm, respectively, (Yamaguchi et al., 1994; Shukla et al., 1997; Buchannan et al., 2000) and the high degree of thermal metamorphism undergone by these samples (Petrologic type 6; Yamaguchi et al., 1996). The temperature-time estimates of metamorphism vary between samples, but the eucrites that experienced the highest degrees of metamorphism are thought to have reached $>1000{ }^{\circ} \mathrm{C}$ or more for $\sim 1$ million years or more (Stolper, 1977; Miyamoto et al., 1985; Miyamoto and Takeda, 1994; Yamaguchi et al., 1994, 1996; Floss et al., 2000; Yamaguchi et al., 2001; Mayne et al., 2009; Yamaguchi et al., 2009; Iizuka et al., 2015) based largely on the extent of major element re-equilibration in pyroxenes and plagioclase. In addition, it is likely that the eucrites experienced short reheating events in the range of $\sim 1050-1100{ }^{\circ} \mathrm{C}$ based on trace element distributions (Floss et al., 2000) and the presence of large tridymite grains and Ti-rich spinels (Yamaguchi et al., 2001; 2009).

To assess the consequences of such metamorphic events on the re-distribution of $\mathrm{Lu}$ and $\mathrm{Hf}$ between pyroxene and its surrounding phases in Millbillillie and Piplia Kalan, we have applied the numerical model developed in this study to the whole-rock pyroxene Lu-Hf ages reported by Bast et al. (2012). The phase surrounding clinopyroxene is assumed to be a partial melt and is treated as a fast-diffusing, fixed-composition matrix. Justification for treating the matrix as a melt comes from an experimental study by Yamaguchi et al. (2013) in which several eucrite samples were heated between 1050 - 
$1100{ }^{\circ} \mathrm{C}$ for 24 hour durations. In experiments run at $1050-1070{ }^{\circ} \mathrm{C}$, small amounts of melt (1-2\%) were distributed throughout pervasive inter-connected networks on preexisting cracks, fractures and grain boundaries of pyroxene without visibly affecting the original petrographic texture at scales of several hundred microns.

At $1050{ }^{\circ} \mathrm{C}$, the Lu diffusion data parallel to the b-axis reported in this study and the Yb data parallel to the c-axis reported by Van Orman et al. (2001) are nearly the same within error (Fig 3; $\mathrm{D}_{\mathrm{Yb} / / \mathrm{c}} \approx 1.73 \times \mathrm{D}_{\mathrm{Lu} / / \mathrm{b}}$ at $\left.1050{ }^{\circ} \mathrm{C}\right)$. Therefore, it is a reasonable simplification to treat Lu diffusion in clinopyroxene as isotropic between $\sim 1000-1050$ ${ }^{\circ} \mathrm{C}$. The simulations presented below utilize the Lu data with //b orientation reported in this study and assume a spherical geometry. Inputting instead the data of Van Orman et al. (2001) would lead to a slightly greater degree of age disruption, but would not produce a significant change in our model results once other variables are taken into account, as discussed below. The initial ${ }^{176} \mathrm{Hf} /{ }^{177} \mathrm{Hf}$ ratio is an input parameter in this model, and the imposed value was 0.27978 as this represents the average initial ${ }^{176} \mathrm{Hf} /{ }^{177} \mathrm{Hf}$ ratio of eucrites that do not exhibit evidence of disrupted internal Lu-Hf systematics (Iizuka et al., 2015b).

Figure $8 \mathrm{~A}$ shows the effect of a $1050{ }^{\circ} \mathrm{C}$ reheating event, sustained for one million years at $4.2 \mathrm{Ga}$, on the bulk $\mathrm{Lu}-\mathrm{Hf}$ isotopic composition of clinopyroxene within the basaltic eucrite Millbillillie (average clinopyroxene radius is $\sim 0.4 \mathrm{~mm}$; Yamaguchi et al., 1994). Millbillillie has a composite texture and variable clinopyroxene grain size, but the average radius should be an appropriate approximation for these simulations since clinopyroxene aliquots were not separated based on grain size. Further closed-system decay/ingrowth after the thermal disturbance leads to a pyroxene-whole-rock age of 
4.957 Ga, which is in excellent agreement with the observed 4-point pyroxene-wholerock Lu-Hf age of $4.94 \pm 0.06 \mathrm{Ga}$ reported by Bast et al. (2012). This model can also explain the 2-point pyroxene-whole-rock Lu-Hf age of $4.9 \pm 0.05 \mathrm{Ga}$ observed for the eucrite Piplia Kalan (Bast et al., 2012), which has a similar average clinopyroxene radius of $\sim 0.5 \mathrm{~mm}$ (Shukla et al., 1997; Buchannan et al., 2000). Running a similar simulation with the slightly coarser grain size of Piplia Kalan yields a Lu-Hf age of $4.872 \mathrm{Ga}$ (Fig. 8B). Although the metamorphic history of the eucrites is somewhat complex, and did not occur as a single thermal pulse as we have assumed in this model (e.g. Steele and Smith, 1976; Metzler et al., 1995; Yamaguchi et al., 1996; Buchannan et al., 2000; Floss et al., 2000; Kleine et al., 2005; Yamaguchi et al., 2009), preferential diffusive loss of Lu occurring throughout multiple shorter heating pulses would have approximately the same net effect as a single thermal event since the $D(t) d t$ values resulting from one or several thermal pulses should be similar. Nonetheless, model results imposing multiple thermal pulses are illustrated in Fig. 9.

Also shown in Figs. 8A and 8B are the ${ }^{176} \mathrm{Hf} /{ }^{177} \mathrm{Hf}$ and ${ }^{176} \mathrm{Lu} /{ }^{177} \mathrm{Hf}$ values of ilmenite, plagioclase and composite aliquots from Millbillillie and Piplia Kalan. These data are considerably scattered, but are qualitatively consistent with loss of Lu to a partial melt phase. Because these aliquots all have ${ }^{176} \mathrm{Lu} /{ }^{177} \mathrm{Hf}$ ratios that are lower than those of the whole rock, loss of Lu would lead to an internal Lu-Hf age that is younger than the crystallization age. It was indeed found by Bast et al. (2012) that a 4-point plagioclasecomposite-whole-rock Lu-Hf isochron from Millbillillie produced an age of $3.70 \pm 0.06$ Ga. Quantitative modeling of this age would require $\mathrm{Lu}$ and $\mathrm{Hf}$ diffusion data in at least plagioclase and ideally ilmenite as well; although there are reported data for REE 
diffusion in plagioclase (Cherniak, 2003), the remaining necessary data, including Hf in plagioclase, are currently unavailable. The triclinic symmetry of plagioclase makes performing such diffusion experiments an extremely difficult and time-consuming task due to the fact that in triclinic phases no principal diffusion directions coincide with the crystallographic axes (Nye, 1957), and we do not consider these data critical for the arguments presented in this paper in light of the quantitative success in modeling the pyroxene-whole-rock ages and qualitative compatibility of the ilmenite and plagioclase data with our model.

Two critical variables in this numerical model are the partition coefficient $(\bigoplus)$ of Lu between clinopyroxene and the surrounding partial melt, and the time at which the reheating event(s) occurred. Figure 10 shows the calculated pyroxene-whole-rock Lu-Hf ages for Millbillillie that result from varying these parameters, as well as the range of experimental partitioning data available in the literature. The corresponding diagram for Piplia Kalan is omitted for brevity since it is nearly identical. The timing of metamorphism was varied between 10 and $554 \mathrm{Myr}$ after igneous crystallization at 4.564 $\mathrm{Ga}$, in order to reflect the range in estimates of the timing of widespread metamorphism on Vesta reported in previous studies (e.g. Prinzhofer et al., 1992; Nyquist et al., 1997; Tera et al., 1997; Bogard and Garrison, 2003; Kleine et al., 2005; Kunz et al., 2005; Dietderich et al., 2013; Iizuka et al., 2015). The global metamorphic event most likely to have caused isotopic disturbance of the eucrites is thought to have occurred prior to 4.2 $\mathrm{Ga}$ based on the Lu-Hf and $\mathrm{Pb}-\mathrm{Pb}$ ages of Jonzac (which were likely fully reset during thermal metamorphism) and high-precision U-Pb zircon dating (Dietderich et al., 2013; Iizuka et al., 2015), although it is possible that widespread metamorphism occurred later 
than 4.2 Ga as well. In addition, Ar degassing of the eucrites via impact events, which occurred later than the global metamorphic event, are well documented (Yamaguchi et al., 2001; Bogard and Garrison, 2003) and would have resulted in minor reheating episodes, though it is unlikely that impact heating would have significantly affected LuHf systematics in pyroxene (Bloch and Ganguly, 2014). Although we have included simulations illustrating that metamorphic episode(s) occurring up to $500 \mathrm{Myr}$ after the igneous crystallization of the eucrites could have produced the observed Lu-Hf ages, simulations that imposed metamorphism before approximately $4.2 \mathrm{Ga}$ are likely a better representation of the thermal history of the eucrites.

4.3 Rationale for using Lu and Hf diffusion data from diopside to model diffusive resetting of pyroxene-whole rock Lu-Hf ages in Millbillillie and Piplia Kalan

Although we have carried out the diffusion experiments reported in this study on diopside, pyroxene in the eucrites Millbillillie and Piplia Kalan is predominantly pigeonite that was partly inverted to orthopyroxene. The inversion, which was accompanied by the development of (001) exsolution lamellae of augites in the host crystal, was suggested to be the result of thermal metamorphism of the eucrites (Yamaguchi et al., 1994).

During thermal metamorphism, Lu and Hf diffusion would have taken place not only in the host pigeonite, but also in orthorpyroxene formed by its inversion as well as in the exsolved augite lamellae. From a crystal structural point of view, the diffusion of a REE in pigeonite is expected to be intermediate between that in diopside and enstatite. 
This is due to the fact that the coordination number of the lattice site that hosts large cations like the trivalent rare earths, M2, changes from 8 in diopside to 7 in pigeonite and 6 in enstatite; however, as shown by Cherniak and Dimanov (2010), the diffusion kinetics of trivalent rare earths in diopside and enstatite, spanning the range of $\mathrm{Eu}-\mathrm{Yb}$ (mass numbers 63-70) are quite similar at $1100{ }^{\circ} \mathrm{C}$, which is close to the inferred temperature of thermal metamorphism of Millbillillie and Piplia Kalan. Lutetium, which has a mass number of 71, should be expected to show similar behavior, and indeed, as illustrated in Fig. 3, $\mathrm{D}(\mathrm{Lu}) / / \mathrm{c}$ determined in this study at $1200{ }^{\circ} \mathrm{C}$ is almost the same as $\mathrm{D}(\mathrm{Yb}) / / \mathrm{c}$ determined by Van Orman et al. (2001). Furthermore, in Fig. 11 we have compared our data with the Nd in orthopyroxene diffusion data of Sano et al. (2011) and the Ti in orthopyroxene diffusion data of Cherniak and Liang (2012). As this comparison clearly shows, there is excellent agreement between our Lu in diopside data, Van Orman et al.'s $\mathrm{Yb}$ in diopside data and Sano et al.'s Nd in orthopyroxene data, in two crystallographic orientations within the ranges of temperature investigated in these studies. Because Ti and $\mathrm{Hf}$ are both tetravalent and have comparable ionic radii ( 0.605 and 0.71 angstroms, respectively), their diffusivities should be quite similar and indeed show good agreement. For the reasons discussed above, we would expect diffusion in diopside and pigeonite to be more similar than that between diopside and enstatite.

Thus, we assume that the diffusivity of $\mathrm{Lu}^{3+}$ in diopside, determined in this study, closely approximates that in pigeonite and orthopyroxene (or inverted pigeonite). In addition, we also assume that the observed disparity between the diffusivities of $\mathrm{D}(\mathrm{Lu})$ and $\mathrm{D}(\mathrm{Hf}$ ) in diopside (Figs. 2 and 3) approximately holds for the other pyroxene phases. This assumption is further justified by the fact that similar disparity between the 
diffusivities of Hf and Lu has also been found in co-diffusion experiments in garnet, following the same experimental and analytical protocols (Bloch et al., 2015).

We would like to remark at this stage that traditional diffusion experiments are not possible on pigeonite for two reasons: First, gem quality homogenous pigeonite crystals of sufficiently large size to be suitable for diffusion experiments are not found in nature. Second, pigeonite would invert to orthopyroxene during the diffusion experiments once the P-T conditions fall below the inversion temperature. The infeasibility of conducting diffusion experiments on pigeonite is exemplified by the fact that to date not one directly measured diffusion datum on pigeonite is available in the literature, although there have been many studies on the diffusion kinetic behavior of major divalent cations and trace elements in pyroxenes, as summarized and discussed by Cherniak and Dimanov (2010).

\subsection{Applicability of existing experimental partitioning data to numerical simulations of Millbillillie and Piplia Kalan}

As illustrated in Fig. 10, the partition coefficients required to produce the observed age of Millbillillie are well within the range of available experimental data (Blundy and Dalton, 2000; Johnston and Schwab, 2004; Dygert et al., 2014; Laubier et al., 2014). With the exception of Dygert et al. (2014), these experimental studies measured $\bigoplus_{Y b}$ and not $\bigoplus_{L u}$; however, due to the identical valence state and nearly identical ionic radii of $\mathrm{Yb}$ and $\mathrm{Lu}$ in 8-fold coordination (Shannon, 1976), the partition coefficients of these species in diopside should not differ significantly (Blundy and 
Wood, 2003). The partitioning experiments described above were run under a range of pressures and temperatures, and used materials that varied in chemical composition. Of these studies, the dataset of Dygert et al. (2014) is probably the most appropriate for the model results presented here due to the fact that the authors used Fe-rich basalts as starting material, which more closely resemble the chemical composition of the eucrites (Kitts and Lodders, 1998) and the partial melt compositions observed by Yamaguchi et al. (2013) than the more Mg-rich starting materials used in the other studies. Furthermore, the temperatures of $1050-1220^{\circ} \mathrm{C}$ imposed by Dygert et al. (2014) are closer to the conditions of metamorphism undergone by the eucrites than the temperatures imposed by Dalton and Blundy (2000), Johnston and Schwab (2004) or Laubier et al. (2014), which were $1375-1640{ }^{\circ} \mathrm{C}, 1375-1640{ }^{\circ} \mathrm{C}$ and $1150-1190{ }^{\circ} \mathrm{C}$, respectively.

Of the partitioning studies described above, only the experiments of Laubier et al. (2014) were conducted at low pressure (0.1 MPa). The experiments of Dalton and Blundy (2000), Johnston and Schwab (2004) and Dygert et al. (2014) were run in the ranges of 0.8-3 GPa, $1 \mathrm{GPa}$ and 0.8-2.2 GPa, respectively. This is a significant point because the thermal metamorphism of the eucrites is believed to have occurred at relatively low pressures (from $\leq 8 \mathrm{~km}$ (Miyamoto and Takeda, 1994) to 15-25 km (Yamaguchi et al., 1997) burial depths) after burial beneath progressive lava flows and subsequent metamorphism via transient heat from the cooling lava and heat from the interior of Vesta (Yamaguchi et al., 1996; 2001; 2009; Mayne et al., 2009). Furthermore, the partial melt phase generated during metamorphism of the eucrites likely had higher P and Ti contents than the melts generated in the experimental partitioning studies discussed here (Yamaguchi et al., 2013). 
The experiments of Yamaguchi et al. (2013) produced partial melts with Lu concentrations of $\sim 0.7 \mathrm{ppm}$, which is low enough to lead to a bulk loss of Lu from pyroxene. Castle (2012) reported $\mathrm{Lu}$ concentrations of $0.36 \pm 0.05,0.45 \pm 0.02,0.43 \pm$ 0.05 and $0.45 \pm 0.03$ for pyroxenes in the eucrites Juvinas, Igdi, Stannern and NWA 6475 , respectively. Taking these values as an approximation of the pyroxene and partial melt $\mathrm{Lu}$ concentrations within Millbillillie and Piplia Kalan during reheating, the range of experimental partition coefficients shown in Fig. 10 would lead to Lu loss.

Although none of the available experimental datasets exactly reflect the metamorphic P-T conditions and composition of the eucrites, the fact remains that all of these datasets, which are the result of experiments run under a wide range of P-T conditions and used starting materials that varied in composition, yield partition coefficients that either overlap or fall close to the $\bigoplus_{L u}$ values required to produce the observed pyroxene-whole-rock Lu-Hf ages of Millbillillie and Piplia Kalan. Although the partition coefficient required in order to produce the observed Lu-Hf age does vary as a function of the initial ${ }^{176} \mathrm{Lu} /{ }^{177} \mathrm{Hf}$ ratio of clinopyroxene, the effect is not great enough to drastically alter the required partition coefficients (Bloch and Ganguly, 2015). As illustrated in Figs. 10A and 10B, changing the initial ${ }^{176} \mathrm{Lu} /{ }^{177} \mathrm{Hf}$ ratio of clinopyroxene by slightly more than a factor of 2 (from 0.18 in Fig. 10A to 0.086 in Fig. 10B) still leads to required partition coefficients that are well within the range of available experimental data.

An important aspect of Fig. 10 is that the uncertainty in both the appropriate $\mathrm{Lu}$ partition coefficient and the time at which metamorphism of Millbillillie and Piplia Kalan occurred prohibits us from concluding that diffusive re-distribution of Lu was solely 
responsible for the Lu-Hf ages of these samples. Uncertainty in the diffusion coefficients of $\mathrm{Lu}$ and $\mathrm{Hf}$ also affects the model results, but is dwarfed by the uncertainty in $\mathrm{Lu}$ partitioning and the timing of metamorphism. Although our model results indicate that preferential mobilization of Lu with respect to $\mathrm{Hf}$ alone is capable, within the uncertainty of the relevant model parameters, of producing the observed ages, based on Fig. 10 it is also possible that the metamorphic event caused only part of the age disruption and that one or more other processes also made a significant contribution. For example, Bast et al. (2016) have argued that the Lu-Hf ages of these and other eucrite and angrite samples is predominantly the result of terrestrial weathering. The possibility of terrestrial weathering is not discussed in detail in this paper, but it is certainly possible based on our model results that another mechanism such as terrestrial weathering, metasomatic fluid infiltration (Jones et al., 2014) or some other as yet unidentified process also contributed to the pre-solar apparent ages of these samples. Nonetheless, the model developed in this study, in conjunction with experimental diffusion and partitioning data, indicates that $\mathrm{Lu}$ re-distribution during metamorphism played at least a significant role in the observed LuHf systematics of Millbillillie and Piplia Kalan.

\subsection{Internal Lu-Hf isochrons of other eucrites and the angrite achondrites}

In addition to Millbillillie and Piplia Kalan, internal Lu-Hf isochrons have been reported for the eucrites Queen Alexandra Range (QUE) 97053, Grosvenor Mountains (GRO) 95533, Elephant Moraine (EET) 87520, Cumulus Hills (CMS) 04049 (Lapen et al., 2015) and Jonzac (Dietderich et al., 2015). As shown in Table 1, of these eucrites 
only QUE 97053 yields a Lu-Hf age that pre-dates Solar System formation. There are several reasons why not all of the eucrites should exhibit the same amount of, if any, thermal disturbance of their internal Lu-Hf systematics. First, as remarked at the top of section 4.2, the eucrites show variable degrees of metamorphism. Samples that remained at relatively low temperatures or underwent faster cooling are not expected to show the same degree of age disruption. It is also possible that the timing of metamorphism varied somewhat between samples. Second, the grain size within eucrites is highly variable. Millbillillie and Piplia Kalan both have particularly small average pyroxene dimensions, which would lead to a greater degree of Lu loss during the metamorphic episode(s).

The internal Lu-Hf isochron of QUE 97053, which yields an age of $4.736 \pm 0.04$ $\mathrm{Ga}$, is more difficult to explain. Both pyroxene and plagioclase from QUE 97053 have higher ${ }^{176} \mathrm{Lu} /{ }^{177} \mathrm{Hf}$ values than the whole-rock aliquot, and based on the old Lu-Hf age retrieved from this sample, are compatible with having lost Lu to a melt phase. However, the ${ }^{176} \mathrm{Hf} /{ }^{177} \mathrm{Hf}$ and ${ }^{176} \mathrm{Lu} /{ }^{177} \mathrm{Hf}$ values of oxide, which has a ${ }^{176} \mathrm{Lu} /{ }^{177} \mathrm{Hf}$ ratio less than that of the whole rock, is also used to define the $4.736 \pm 0.04 \mathrm{Ga}$ isochron, which is virtually free of scatter (Fig. 1 in Lapen et al., 2015). If a melt phase were present during metamorphism of QUE 97053, then we would expect to see a similar Lu-Hf trend in oxides to what is observed for Millbillillie and Piplia Kalan. A similar problem is encountered for the angrite SAH 99555, as described in Table 1. This sample produced a statistically significant pyroxene-whole-rock-olivine Lu-Hf isochron age that yields an age of $4.869 \pm 0.034 \mathrm{Ga}$ (Bizzarro et al., 2012).

Debaille et al. (2011) suggested that the old age of SAH 99555 could be due to diffusive exchange of $\mathrm{Hf}$ between phosphates and the other constituent minerals of this 
sample. Phosphate is present in both the eucrites and angrites and has a much higher ${ }^{176} \mathrm{Lu} /{ }^{177} \mathrm{Hf}$ ratio, and therefore a far more radiogenic $\mathrm{Hf}$ isotopic signature, than the phases used to construct the internal Lu-Hf isochrons listed in Table 1. As a result, any exchange of $\mathrm{Hf}$ between phosphate and the minerals used to construct the $\mathrm{Lu}-\mathrm{Hf}$ isochrons would result in these phases becoming more radiogenic with respect to their $\mathrm{Hf}$ contents. Such diffusive exchange would effect the Hf isotopic composition of low-Hf phases such as olivine most substantially. Because clinopyroxene has both a higher Hf concentration and a lower $\mathrm{Lu} / \mathrm{Hf}$ ratio than olivine, the authors have argued that the net result would be an increase in the slope of the $\mathrm{Lu}-\mathrm{Hf}$ isochron and a resulting apparent age that is erroneously old, while still producing a statistically significant apparent isochron. This same line of reasoning can be applied to QUE 97053, provided there was no melt phase present during metamorphism of these samples.

Although diffusive exchange with phosphate would increase the ${ }^{176} \mathrm{Hf} /{ }^{177} \mathrm{Hf}$ ratios of the other constituent minerals, it is not entirely clear that such exchange would lead to an increased isochron slope since diffusive exchange of Lu would occur at the same time. If partitioning between these phases is such that there is a net diffusive flux of $\mathrm{Lu}$ out of phosphate, then the slope of the Lu-Hf isochron may actually decrease. This is especially true in light of the fact that Lu most likely diffuses faster than $\mathrm{Hf}$ in most, if not all, of the constituent minerals of the eucrites and angrites. Regardless of the partitioning behavior of $\mathrm{Lu}$, it seems unlikely that such diffusive re-distribution of $\mathrm{Lu}$ and $\mathrm{Hf}$ would lead to rotated Lu-Hf isochrons that maintain statistically significant linearity. Furthermore, despite the fact that CMS 04049 has a higher equilibrated pyroxene temperature than QUE 97053 ( 880 and $800{ }^{\circ} \mathrm{C}$, respectively; Righter and Lapen, 2010), a pre-solar Lu- 
Hf age is only observed for the latter sample. Because of the similar texture and mineralogy between these samples, if diffusion from phosphate were responsible for the Lu-Hf systematics of QUE 97053, then there is no obvious reason why the same general trends should not be observed in CMS 04049.

In order to quantitatively model diffusive exchange between phosphate and the remaining constituent phases, data for $\mathrm{Lu}$ and $\mathrm{Hf}$ diffusion in all of the involved phases, as well as partitioning data for Lu between phosphate and the other phases, would be required. Alternatively, as pointed out by Debaille (2011) this mechanism predicts a correspondingly reduced phosphate Lu-Hf age in samples exhibiting apparent excess ${ }^{176} \mathrm{Hf}$. Although such analyses are notoriously difficult based on the small grain size of phosphates in these samples, measuring the Lu-Hf age of phosphate would provide a straightforward test for this hypothesis. However, this would only provide a permissive criterion. The presence of a syn- or pre-solar Lu-Hf age in phosphate would contradict this explanation, whereas the presence of a younger age does not outright confirm this hypothesis for the reasons discussed above. Similar Lu-Hf analyses of phosphates were performed on the primitive achondrite Acapulco and the ordinary H5 chondrite Richardton by Amelin (2005) and should be possible on eucrites and angrites in the future.

It must be noted as part of this discussion that Bast et al. (2013) reported internal $\mathrm{Lu}-\mathrm{Hf}$ isochrons that yield ages of $4.760 \pm 0.064$ and $4.635 \pm 0.019 \mathrm{Ga}$ for the angrites D’Orbigny and Northwest Africa (NWA) 4801, respectively. We have not included these results in Table 1 because Sanborn et al. (2015) have reported Lu-Hf ages for these samples more recently and in peer-reviewed format, and because Bast et al. (2013) do not 
list the phases used to construct these isochrons. As noted in Table 1, Sanborn et al. (2015) reported internal Lu-Hf ages for these samples that are the same within error as the accepted age of Solar System formation.

Jonzac is the only eucrite that produces a statistically significant multiphase (oxide, plagioclase, whole rock and pyroxene) internal Lu-Hf isochron that yields a significantly younger date $(4.236 \pm 0.026 \mathrm{Ga}$; Dietderich et al., 2013$)$ than the accepted age of Solar System formation. It is likely that this sample experienced especially prolonged and/or high-temperature metamorphism (petrologic type 7; Yamaguchi et al., 1996) that resulted in full resetting of the Lu-Hf chronometer. Despite the fast diffusion of $\mathrm{Lu}$ with respect to $\mathrm{Hf}$, if the effective diffusion distance of $\mathrm{Hf}$ during a thermal event (given by $D(t) d t$ ) is greater than the grain size of the phase of interest, then diffusive resetting of $\mathrm{Hf}$ isotopes between the constituent phases will offset uptake or loss of Lu, and the Lu-Hf chronometer will be effectively reset as described in section 4.1. This possibility is in line with the interpretations of Dietderich et al. (2013), who suggest that the Lu-Hf age of Jonzac reflects metamorphic disturbance(s) between $\sim 4.2-4.3 \mathrm{Ga}$. If the Lu-Hf chronometer was fully reset by metamorphism at $\sim 4.2-4.3 \mathrm{Ga}$, then the Sm$\mathrm{Nd}$ age retrieved from the same aliquots should also reflect this metamorphic event; however, no Sm-Nd data were reported for Jonzac.

\section{Conclusions}

We have reported new diffusion data for $\mathrm{Lu}$ in diopside, and used these data in conjunction with the Hf diffusion data reported by Bloch et al. (2014) to illustrate the effects of initial cooling and subsequent thermal metamorphism on the Lu-Hf systematics 
of clinopyroxene. We find that the age disruption caused during initial cooling is likely to be negligible, and that the Lu-Hf systematics of clinopyroxene should closely coincide with the closure temperature of $\mathrm{Hf}$ in most samples that did not undergo subsequent reheating. For samples that did undergo substantial reheating events, diffusive loss or gain of Lu has the capacity to significantly disrupt Lu-Hf systematics within clinopyroxene. The extent to which the Lu-Hf age recorded by clinopyroxene is affected by reheating varies as a function of the temperature, timing and duration of the reheating episode, the characteristic grain size and the partitioning of Lu between clinopyroxene and its adjacent phase(s).

We have used the model developed in this study, in conjunction with available experimental Lu partitioning data between clinopyroxene and silicate melts, to simulate the effect of reheating on the Lu-Hf systematics of the eucrites Millbillillie and Piplia Kalan. We find that the $\sim 4.9$ Ga pyroxene-whole-rock Lu-Hf ages of the eucrites Millbillillie and Piplia Kalan can be well explained by diffusive loss of Lu from the constituent phases to a partial melt during high-temperature metamorphism; however, the Lu-Hf systematics of the eucrites and angrites vary substantially between samples and our model does not offer a comprehensive solution to the problem of excess ${ }^{176} \mathrm{Hf}$ in these meteorites. Although the compatibility of Millbillillie and Piplia Kalan with our model does not rule out that other mechanisms could have contributed to the observed Lu-Hf systematics of these samples, these findings do illustrate the potential impact that reheating can have on Lu-Hf ages. As such, when interpreting Lu-Hf isochrons the possibility of diffusive re-distribution of $\mathrm{Lu}$ and $\mathrm{Hf}$ should be carefully considered on a case-by-case basis. 


\section{Acknowledgements:}

This research was supported by NASA Cosmochemistry grant No. NNX10A179 to JG, which is gratefully acknowledged. Thanks are due to Jon Patchett and Mauricio Ibañez-Mejia for useful discussions, and in particular to Richard Hervig for SIMS analyses of our experimental products at Arizona State University. Thoughtful reviews by Thomas Lapen, Danielle Cherniak and one anonymous reviewer led to a much-improved final version of the paper. Finally, we are grateful to Yuri Amelin and Marc Norman for editorial handling as well as thoughtful suggestions for improving this manuscript. 


\section{References}

Albarède F., Scherer E., Blichert-Toft J., Rosing M., Simionovici A. and Bizzarro M. (2006) Gamma-ray irradiation in the early Solar System and the conundrum of the

${ }^{176}$ Lu decay constant. Geochim. Cosmochim. Acta 70, 1261-1270.

Amelin, Y. (2005) Meteorite phosphates show constant ${ }^{176} \mathrm{Lu}$ decay rate since 4557 million years ago. Science 310, 839-841.

Amelin Y., Irving A. (2007) Seven million years of evolution on the angrite parent body from $\mathrm{Pb}$-isotopic data. Workshop on Chronology of Meteorites and the Early Solar System: 1374.

Amelin Y. (2008) U-Pb ages of angrites. Geochim. Cosmochim. Acta 72: 221-232.

Amelin Y., Wimpenny J. and Yin Q-Z (2011a) Angrite Lu-Hf whole rock data provide no direct support to accelerated decay of ${ }^{176} \mathrm{Lu}$ by supernova irradiation. Workshop on Formation of the First Solids in the Solar System, abstr. 9014.

Amelin Y., Kaltenbach A., Stirling C. (2011b) The U-Pb systematics and cooling rate of plutonic angrite NWA 4590. Lunar Planet. Sci. Conf. XLII, abstr. 2879.

Bast R., Scherer E., Taetz S., Sprung P., Mezger K. and Srinivasan G. (2012) Internal LuHf isotope systematics of the eucrites Millbillillie and Piplia Kalan. Lunar Planet. Sci. Conf. XLII, abstr. 2542.

Bast R., Scherer E., Mezger K., Fischer-Gödde M. and Sprung P. (2013) Internal Lu-Hf isotope systematics of the quenched angrite D'Orbigny and two plutonic angrites. Mineralogical Magazine 77(5), 665.

Bast R., Scherer E., Sprung P. and Mezger K. (2016) Evidence for recent disturbance of the ${ }^{176} \mathrm{Lu}^{-176} \mathrm{Hf}$ system in meteorites. Goldschmit Conf. Abstr. \#162

Binzel R. and Xu S. (1993) Chips off of asteroid Vesta: Evidence for the parent body of basaltic achondrite meteorites. Science 260, 186-191.

Bizzarro M., Baker J., Haack H., Ulfbeck D. and Rosing M. (2003) Early history of Earth's crust-mantle system inferred from hafnium isotopes in chondrites. Nature 421, 931-933.

Bizzarro, M., Connelly, J. N., Thrane, K. and Borg, L. E. (2012) Excess hafnium-176 in meteorites and the early Earth zircon record. Geochem. Geophys. Geosys. 13, QO3002. Doi:10.1029/2011GC004003.

Blichert-Toft J., Boyet M., Télouk P. and Albarède F. (2002) ${ }^{147} \mathrm{Sm}_{-}{ }^{143} \mathrm{Nd}$ and ${ }^{176} \mathrm{Lu}-{ }^{176} \mathrm{Hf}$ 
in eucrites and the differentiation of the HED parent body. Earth Planet. Sci. Lett. 204, 167-181.

Bloch E. and Ganguly J. (2014) ${ }^{176} \mathrm{Lu}^{-}{ }^{176} \mathrm{Hf}$ and ${ }^{147} \mathrm{Sm}_{-}{ }^{143} \mathrm{Nd}$ ages of the Martian shergottites: Evaluation of the shock-resetting hypothesis through diffusion kinetic experiments and modeling, and petrological observations. Earth Planet. Sci. Lett. 395, 173-183.

Bloch E., Ganguly J., Hervig R. and Cheng W. (2015) ${ }^{176} \mathrm{Lu}-{ }^{176} \mathrm{Hf}$ geochronology of garnet I: Experimental determination of the diffusion kinetics of $\mathrm{Lu}^{3+}$ and $\mathrm{Hf}^{4+}$ in garnet, closure temperatures and geochronological implications. Contrib. Mineral. Petrol. 169, 14.

Bloch E. and Ganguly J. (2015) ${ }^{176} \mathrm{Lu}-{ }^{176} \mathrm{Hf}$ geochronology of garnet II: Numerical simulations of the development of garnet-whole-rock ${ }^{176} \mathrm{Lu}-{ }^{176} \mathrm{Hf}$ isochrons and a new method for constraining the thermal history of metamorphic rocks. Contrib. Mineral. Petrol. 169, 14.

Blundy J. and Dalton J. (2000) Experimental comparison of trace element partitioning between clinopyroxene and melt in carbonate and silicate systems, and implications for mantle metasomatism. Contrib. Mineral. Petrol. 139, 356-371.

Blundy J. and Wood B. (2003) Partitioning of trace elements between crystals and melts. Earth Planet. Sci. Lett. 210, 383-397.

Bogard D. and Garrison D. (1995) ${ }^{39} \mathrm{Ar}-{ }^{40} \mathrm{Ar}$ age of the eucrite and constraints on the time of pyroxene equilibration. Geochim. Cosmochim. Acta 59, 4317-4322.

Bogard D. and Garrison D. (2003) ${ }^{39} \mathrm{Ar}-{ }^{40} \mathrm{Ar}$ ages of eucrites and thermal history of asteroid 4 Vest. Met. Planet. Sci. 38, 669-710.

Bouvier A., Vervoort J. and Patchett J. (2008) The Lu-Hf and Sm-Nd isotopic composition of CHUR: Constraints from unequilibrated chondrites and implications for the bulk composition of terrestrial planets. Earth Planet. Sci. Lett. 273, 48-57.

Bouvier A., Brennecka G., Sanborn M., Wadhwa M. (2011) U-Pb chronology of a newly recovered angrite. Lunar Planet. Sci. Conf. XLII, abstr. 2747.

Bouvier A., Blichert-Toft J., Boyet M., Albarède F. (2015) ${ }^{147} \mathrm{Sm}^{-}{ }^{143} \mathrm{Nd}$ and ${ }^{176} \mathrm{Lu}^{-177} \mathrm{Hf}$ systematics of eucrite and angrite meteorites. Met. Planet. Sci. 50, 1896-1911.

Boyet M., Carlson R. and Horan M. (2010) Old Sm-Nd ages for cumulate eucrites and redetermination of the solar system initial ${ }^{146} \mathrm{Sm} /{ }^{144} \mathrm{Sm}$ ratio. Earth Planet. Sci. Lett. 291, 172-181. 
Brennecka G. and Wadhwa M., (2012) Uranium isotope compositions of the basaltic angrite meteorites and the chronological implications for the early solar system. Proc. Nat. Acad. Sci. USA 109, 9299-9303.

Buchannan P., Mittlefehldt D., Hutchison R., Koeberl C., Lindstrom D. and Pandit M. (2000) Petrology of the Indian eucrite Piplia Kalan. Met. Planet. Sci. 35, 609-615.

Castle N. (2012) Pyroxene chemistry in polymict eucrite Northwest Africa 6475: Contrasts with Juvinas, Stannern and Igdi, and evaluation of models for eucrite magma evolution. Ph.D. Thesis, University of Washinton, USA.

Cherniak D. (2003) REE diffusion in feldspar. Chem. Geol. 193, 25-41.

Cherniak D., Dimanov A. (2010) Diffusion in pyroxene, mica and amphibole. Rev. Mineral. Geochem. 72, 409-446.

Cherniak D., Liang Y. (2012) Ti diffusion in natural pyroxene. Geochim. Cosmochim. Acta 98, 31-47.

Crank, J. (1975) The Mathematics of Diffusion. Oxford Press.

Debaille V., Yin Q-Z and Amelin Y. (2011) The role of phosphates for the Lu-Hf chronology of meteorites. Formation of the First Solidas in the Solar System. Lunar Planet. Inst., Houston. Abstr. 9066.

Dietderich J., Lapen T., Andreasen R. and Righter M. (2013) Isotope systematics of the type 7 eucrite Jonzac: A look into the history of the eucrite parent body using the lutetium-hafnium, lead-lead, \& uranium-lead isotopic systems. Lunar Planet. Sci. Conf. XLIV, abstr. 2879.

Dodson M. (1973) Closure temperatures in cooling geochronological and petrological systems. Contrib. Mineral. Petrol. 40, 259-275.

Dygert N., Liang Y., Sun C. and Hess P. (2014) An experimental study of trace element partitioning between augite and Fe-rich basalts. Geochim. Cosmochim. Acta 132, $170-186$.

Floss C., Crozaz G., Yamaguchi A. and Keil K. (2000) Trace element constraints on the origins of highly metamorphosed Antarctic eucrites. Antarct. Met. Res. 13, 222237.

Ganguly J., Tirone M. and Hervig R. (1998) Diffusion kinetics of samarium and neodymium in garnet, and a method for determining cooling rates of rocks. Science 281, 805-807.

Ganguly J., Tirone M. (1999) Diffusion closure temperature and age of a mineral with 
arbitrary extent of diffusion: Theoretical formulation and applications. Earth Planet. Sci. Lett. 170, 901-909.

Hopkins M., Mojzsis S., Bottke W., Abramov O. (2015) Micrometer-scale U-Pb age domains in eucrite zircons, impact re-setting, and the thermal history of the HED parent body. Icarus 245: 367-378.

Iizuka T., Yamaguchi A., Haba M., Amelin Y., Holden P., Zink S., Huyskens M. and Ireland T. (2015a) Timing of global crustal metamorphism on Vesta as revealed by high-precision $\mathrm{U}-\mathrm{Pb}$ dating and trace element chemistry of eucrite zircon. Earth Planet. Sci. Lett. 409, 182-192.

Iizuka T., Yamaguchi T., Hibiya Y. and Amelin Y. (2015b) Meteorite zircon constraints on the bulk Lu-Hf isotope composition and early differentiation of the Earth. Proc. Nat. Acad. Sci. USA 112, 5331-5336.

Jacobsen S. and Wasserburg G. (1984) Sm-Nd isotopic evolution of chondrites and achondrites, II. Earth Planet. Sci. Lett. 67, 137-150.

James F. and Roos M. (1975) MINUIT, a system of function minimization and analysis of the parameter errors and correlations. Comput. Phys. 10, 343-347.

Johnston D. and Schwab B. (2004) Constraints on clinopyroxene/melt partitioning of $\mathrm{REE}, \mathrm{Rb}, \mathrm{Sr}, \mathrm{Ti}, \mathrm{Cr}, \mathrm{Zr}$, and $\mathrm{Nb}$ during mantle melting: First insights from direct peridotite melting experiments at $1.0 \mathrm{GPa}$. Geochim. Cosmochim. Acta 268, 49494962.

Jones R., McCubbin F., Dreeland L., Guan Y., Burger P., Chearer C. (2014) Phosphate minerals in LL chondrites: A record of the action of fluids during metamorphism on ordinary chondrite bodies. Geochim. Cosmochim. Acta 132, 120-140.

Kelly E., Carlson W. and Connelly J. (2011) Implications of garnet resorption for the Lu Hf geochronometer: An example from the contact aureole of the Makhavinekh Lake Pluton, Labrador. J. Met. Geol. 29, 901-916.

Kitts K. and Lodders K. (1998) Survey and evaluation of eucrite bulk compositions. Met. Planet. Sci. 33, A197-A213.

Kleine T., Mezger K., Palme H., Scherer D. and Münker C. (2005) The W isotopic composition of eucrite metals: Constraints on the timing and cause of the thermal metamorphism of basaltic eucrites. Earth Planet Sci.Lett. 231, 41-52.

Kleine T., Hans U., Irving A. and Bourdon B. (2012) Chronology of the angrite parent body and implications for core formation in protoplanets. Geochim. Cosmochim. Acta 84, 186-203. 
Kohn M. (2009) Models of garnet differential geochronology. Geochim. Cosmochim. Acta 73, 170-182.

Kunz J., Trieloff M., Bobe K., Metzler K., Stöffler D. and Jossberger E. (1995) The collisional history of the HED parent body inferred from ${ }^{40} \mathrm{Ar}-{ }^{39} \mathrm{Ar}$ ages of eucrites. Planet. Space Sci. 43, 527-543.

Lapen T., Righter M. and Andreasen R. (2015) Lu-Hf and Sm-Nd isotope systematics of non-cumulate eucrites. Lunar Planet. Sci. Conf. XLVI, abstr. 2863.

Lasaga S. (1998) Kinetic theory in earth sciences. Princeton Press.

Laubier M., Grove T. and Langmuir C. (2014) Trace element partitioning for basaltic and basaltic andesitic melts: An experimental and laser ICP-MS study with application to the oxidation state of mantle source regions. Earth Planet Sci. Lett. 392, 265-278.

Lugmair G. and Galer S. (1992) Age and isotopic relationship among the angrites Lewis Cliff 86010 and Angra do Reis. Geochim. Cosmochim. Acta 56, 1673-1694.

Lugmair G. and Shukolyukov A. (1998) Early solar system timescales according to ${ }^{53} \mathrm{Mn}-$ ${ }^{53}$ Cr systematics. Geochim. Cosmochim. Acta 62, 2863-2886.

Manhes, G., Allegre, C., Provost, A. (1984) U-Th-Pb systematics of the eucrite "Juvinas": Precise age determination and evidence for exotic lead. Geochim. Cosmochim. Acta 48: 2247-2264.

Markowski A., Quitté G., Kleine T., Halliday A., Bizzarro M. and Irving A. (2007) Hafnium-tungsten chronometry of angrites and the earliest evolution of planetary objects. Earth Planet. Sci. Lett. 262, 214-229.

Martin C., Debaille V., Lanari P., Goderis S., Vandendael I., Vanhaecke F., Vidal O. and Claeys P. (2013) REE and Hf distributions among mineral phases in the CV-CK clan: A way to explain present-day $\mathrm{Hf}$ isotopic variations in chondrites. Geochim. Cosmochim. Acta 120, 496-513.

Mayne R., McSween Jr. H., McCoy T. and Gale A. (2009) Petrology of the unbrecciated eucrites. Geochim. Cosmochim. Acta 73, 794-819.

Metzler K., Bobe K., Palme H., Spettel B. and Stöffler D. (1995) Thermal and impact metamorphism of the HED-asteroid. Planet. Space Sci. 43, 499-525.

Misawa K., Yamaguchi A., Kaiden H. (2005) U-Pb and ${ }^{207} \mathrm{~Pb}-{ }^{206} \mathrm{~Pb}$ ages of zircons from basaltic eucrites: Implications for early basaltic volcanism on the eucrite parent body. Geochim. Cosmochim. Acta 69: 5847-5861. 
Miyamoto M., Duke M. and McKay D. (1985) Chemical zoning and homogenization of pasamonte-type pyroxene and their bearing on thermal metamorphism of a howardite parent body. J. Geophys. Res. 90 (Supplement), C629-C635.

Miyamoto M. and Takeda H. (1994) Evidence for excavation of deep crustal material of a Vesta-like body from Ca compositional gradients in pyroxene. Earth Planet. Sci. Lett. 122, 343-349.

Nye J. (1957) Physical Properties of Crystals. Oxford Press.

Nyquist L., Bansal B., Wiesmann H. and Shih C-Y (1994) Neodymium, strontium, and chromium isotopic studies of the LEW 86010 and Angra del Reis meteorites and the chronology of the angrite parent body. Meteoritics 29, 872-885.

Nyquist L., Bogard D., Takeda H., Bansal B., Wiessmann H. and Shih C-Y (1997) Crystallization, recrystallization, and impact-metamorphic ages of eucrites Y792510 and Y791186. Geochim. Cosmochim. Acta 61, 2119-2138.

Patchett J. and Tatsumoto, M. (1980) Lu-Hf total-rock isochron for the eucrite meteorites. Nature 288, 571-574.

Patchett J., Vervoort J., Söderlund U. and Salters V. (2004) Lu-Hf and Sm-Nd isotopic systematics in chondrites and their constraints on the Lu-Hf properties of the Earth. Earth Planet. Sci. Lett. 222, 29-41.

Righter M., Lapen T. (2010) Petrology, mineralogy and mineral chemistry of Antarctic monomict eucrites CMS 04049 and QUE 97053. Lunar. Planet. Sci. Conf. XLI, abstr. 2629.

Righter M., Shaulis B., Lapen T. (2012) U-Pb and ${ }^{207} \mathrm{~Pb}^{206} \mathrm{~Pb}$ age of zircons from polymict eucrites and howardites. Lunar. Planet. Sci. Conf. XLIII, abstr. 2562.

Prinzhofer A., Papanastassiou D. and Wasserburg G. (1992) Samarium-neodymium evolution of meteorites. Geochim. Cosmochim. Acta 56, 797-815.

Sanborn M., Carlson R. and Wadhwa M. (2015) ${ }^{147,146} \mathrm{Sm}^{-143,142} \mathrm{Nd},{ }^{176} \mathrm{Lu}-{ }^{176} \mathrm{Hf}$, and ${ }^{87} \mathrm{Rb}^{87} \mathrm{Sr}$ Systematics in the Angrites: Implications for Chronology and Processes on the Angrite Parent Body. Geochim. Cosmochim. Acta. doi: http//dx.doi.org/10.1016/j.gca.2015.08.026.

Sano J., Ganguly J., Hervig R., Dohmen R. and Zhang X. (2011) Neodymium diffusion in orthopyroxene: Experimental studies and applications to geologic and planetary problems Geochim. Cosmochim. Acta 75, 4684-4698.

Scherer E. Münker C. and Mezger K. (2001) Calibration of the lutetium-hafnium clock. Science 293, 683-687. 
Shannon R. (1976) Revised effective ionic radii and systematic studies of interatomic distances in halides and chalcogenides. Acta Cryst. A32, 751-767.

Shukla A., Shukla P., Suthar K., Bhandari N., Vaya V., Sisodia M., Sinha Roy S., Rao K. and Rajawat R. (1997) Piplia Kalan eucrite: Fall, petrography and chemical characteristics. Met. Planet. Sci. 32, 611-615.

Shukolyukov A. and Begemann F. (1996) Pu-Xe dating of eucrites. Geochim. Cosmochim. Acta 60, 2453-2471.

Shukolyukov A. and Lugmair G. (2008) Mn-Cr chronology of eucrite CMS 04049 and angrite NWA 2999. Lunar. Planet. Sci. Conf. XXXIX, abstr. 2094.

Smoliar M. (1993) A survey of Rb-Sr systematics of eucrites. Meteoritics 28, 105-113.

Sneeringer M., Hart S., Shimizu N. (1984) Strontium and samarium diffusion in diopside. Geochim. Cosmochim. Acta 48, 1589-1608.

Söderlund U., Patchett J., Vervoort J. and Isachsen C. (2004) The ${ }^{176}$ Lu decay constant determined by $\mathrm{Lu}-\mathrm{Hf}$ and $\mathrm{U}-\mathrm{Pb}$ isotope systematics of Precambrian mafic intrusions. Earth Planet. Sci. Lett. 219, 311-324.

Spivak-Birndorf L., Wadhwa M., and Janney P. (2009) ${ }^{26} \mathrm{Al}-{ }^{26} \mathrm{Mg}$ systematics in D'Orbigny and Sahara 99555 angrites: Implications for high-resolution chronology using extinct chronometers. Geochim. Cosmochim. Acta 73, 52025211.

Sprung P. Scherer E. Upajhyay D., Leya I. and Mezger K. (2010) Non-nucleosynthetic heterogeneity in non-radiogenic stable Hf isotopes: Implications for early solar system chronology. Earth Planet. Sci. Lett. 295, 1-11.

Sprung P., Kleine T. and Scherer E. (2013) Isotopic evidence for chondritic Lu/Hf and $\mathrm{Sm} / \mathrm{Nd}$ of the moon. Earth Planet. Sci. Lett. 380, 77-87.

Steele I. and Smith J. (1976) Mineralogy of the Ibitra eucrite and comparison with other eucrites and lunar samples. Earth Planet. Sci. Lett. 33, 67-78.

Stolper E. (1977) Experimental petrology of the eucrite meteorites. Geochim. Cosmochim. Acta 41, 587-611.

Tera F., Carlson R. and Boctor N. (1997) Radiometric ages of basaltic achondrites and their relation to the early history of the solar system. Geochim. Cosmochim. Acta 61, 1713-1731.

Thrane K., Connelly J., Bizzarro M., Meyer B. and The L-H. (2010) Origin of excess 
${ }^{176} \mathrm{Hf}$ in meteorites. Astrophys. J. 717, 861-867.

Tirone M., Ganguly J., Dohmen R., Langenhorst F., Hervig R. and Becker H-W (2005) Rare earth diffusion kinetics in garnet: Experimental studies and applications. Geochim. Cosmochim. Acta 69, 2385-2398.

Van Orman J., Grove T. and Nobumichi S. (2001) Rare earth element diffusion in diopside: Influence of temperature, pressure, and ionic radius, and an elastic model for diffusion in silicates. Contrib. Mineral. Petrol. 141, 687-703.

Williams P. and Baker J. (1981) Implantation and ion beam mixing in thin film analysis. Nuc. Inst. Meth. 182/183, 15-24.

Winchell P. (1969) The compensation law for diffusion in silicates. High Temp. Sci. 1, 200-215.

Wimpenny, J., Amelin, Y., Yin, Q-Z (2015) The Lu isotopic composition of achondrites: Closing the case for accelerated decay of ${ }^{176}$ Lu. Astrophys. J. Lett. 812: L3.

Yamaguchi, A., Takeda, H., Bogard, D. and Garrison, D. (1994) Textural variations and impact history of the Millbillillie eucrite. Meteoritics 29, 237-245.

Yamaguchi A., Taylor J. and Keil K. (1996) Global crustal metamorphism of the eucrite parent body. Icarus 124, 97-112.

Yamaguchi A., Taylor J. and Keil K. (1997) Metamorphic history of the eucritic crust of 4 Vesta. J. Geophys. Res. 102, 13,381-13,386.

Yamaguchi A., Taylor J., Keil K., Floss C., Crozaz G., Nyquist L., Bogard D., Garrison D., Reese Y., Wiesmann H. and Shih C-Y (2001) Post-crystallization reheating and partial melting of eucrite EET 90020 by impact into the hot crust of asteroid 4Vesta 4.50 Ga ago. Geochim. Cosmochim. Acta 65, 3577-3599.

Yamaguchi A., Barrat J., Greenwood R., Shirai N., Okamoto C., Setoyanagi T., Ebihara M., Franchi I. and Bohn M. (2009) Crustal partial melting on Vesta: Evidence from highly metamorphosed eucrites. Geochim. Cosmochim. Acta 73, 7162-7182.

Yamguchi A., Mikouchi T., Ito M., Shirai N., Barrat J., Messenger M. and Ebihara M. (2013) Experimental evidence of fast transport of trace elements in planetary basaltic crusts by high temperature metamorphism. Earth Planet. Sci. Lett. 368, 101-109.

Yao L., Liang Y. (2015) Closure temperature in cooling bi-mineralic systems: I. Definition and with application to REE-in-two-pyroxene thermometer. Geochim. Cosmochim. Acta 162, 137-150. 
Zhang X-Y, Ganguly J. and Ito M. (2010) Ca-Mg diffusion in diopside: Tracer and chemical inter-diffusion coefficients. Contrib. Mineral. Petrol. 159, 175-186.

Zhou, Q., Yin, Q-Z, Young, E., Li, X-H, Wu, F-Y, Li, Q-L, Liu, Y., Tang, G-C. (2013) SIMS $\mathrm{Pb}-\mathrm{Pb}$ and $\mathrm{U}-\mathrm{Pb}$ age determination of eucrite zircons at $<5 \mu \mathrm{m}$ scale and the first $50 \mathrm{Ma}$ of the thermal history of Vesta. Geochim. Cosmochim. Acta 110: $152-175$. 


\begin{tabular}{ccccc}
\hline Sample Name & Type & Lu-Hf Age (Ga) & Phases used in isochron & Source \\
\hline Millbillillie & EUC & $4.94 \pm 0.06$ & WR, pyx & Bast et al., 2012 \\
Millbillillie & EUC & $3.7 \pm 0.06$ & Plag, comp, WR & Bast et al., 2012 \\
Piplia Kalan & EUC & $4.9 \pm 0.05$ & Wr, pyx & Bast et al., 2012 \\
QUE 97053 & EUC & $4.736 \pm 0.04$ & Ox, WR, dirty plag, pyx & Lapen et al., 2015 \\
GRO 95533 & EUC & $4.489 \pm 0.025$ & Ox, plag, WR, pyx & Lapen et al., 2015 \\
EET 87520 & EUC & $4.557 \pm 0.017$ & Ox, plag, WR, pyx & Lapen et al., 2015 \\
CMS 04049 & EUC & $4.545 \pm 0.025$ & Ox, plag, pyx & Lapen et al., 2015 \\
Jonzac & EUC & $4.236 \pm 0.026$ & Ox, WR, plag, pyx & Dietderich et al., 2013 \\
SAH 99555 & ANG & $4.869 \pm 0.034$ & Pyx, WR, ol & Bizarro et al., 2012 \\
NWA 4590 & ANG & $4.523 \pm 0.024$ & Pyx, WR, plag, F, ol & Sanborn et al., 2015 \\
NWA 4801 & ANG & $4.563 \pm 0.050$ & Pyx, plag, WR, F, ol & Sanborn et al., 2015 \\
D'Orbigny & ANG & $4.51 \pm 0.097$ & Pyx, WR, plag, F, ol & Sanborn et al., 2015 \\
\hline
\end{tabular}

Table 1: Internal isochron ages of eucrite and angrite meteorites. EUC = eucrite, ANG = angrite. Phases used in isochrons are listed in order of increasing ${ }^{176} \mathrm{Lu} /{ }^{176} \mathrm{Hf}$ ratio. Comp = composite aliquot, $\mathrm{F}=$ fine-grained aliquot ox $=$ oxide, plag = plagioclase, pyx $=$ pyroxene, $\mathrm{WR}=$ whole rock. 


\begin{tabular}{|c|c|c|c|c|c|}
\hline Sample & Orientation & $\mathbf{T}\left({ }^{\circ} \mathbf{C}\right)$ & $\begin{array}{c}\text { Time } \\
\text { (hours) }\end{array}$ & $\begin{array}{c}\operatorname{logDHf}(2 \sigma) \\
\left(\mathrm{m}^{2} / \mathbf{s}\right)\end{array}$ & $\begin{array}{c}\operatorname{logDLu}(2 \sigma) \\
\left(\mathrm{m}^{2} / \mathrm{s}\right)\end{array}$ \\
\hline Dib_mix2 & $/ / \mathrm{b}$ & 1150 & 165.9 & $-20.84(0.112)$ & $-20.51(0.105)$ \\
\hline Dib_Lu3 & $/ / \mathrm{b}$ & 1150 & 165.9 & - & $-20.41(0.091)$ \\
\hline Dib_mix3 & $/ / \mathrm{b}$ & 1150 & 285.3 & $-20.89(0.14)$ & $-20.51(0.086)$ \\
\hline Dib_mix4 & $/ / \mathrm{b}$ & 1150 & 429.1 & $-20.89(0.354)$ & $-20.59(0.087)$ \\
\hline Dib_WHf1 & $/ / \mathrm{b}$ & 1200 & 117.8 & $-20.64(0.124)$ & $-20.32(0.07)$ \\
\hline Dic_mix1 & $/ / \mathrm{c}$ & 1200 & 91.7 & $-20.50(0.082)$ & $-19.46(0.07)^{*}$ \\
\hline Dia_mix1 & $/ / \mathrm{a}$ & 1200 & 91.7 & $-20.62(0.072)$ & $-20.25(0.063)$ \\
\hline Dib_mix5 & $/ / \mathrm{b}$ & 1225 & 95.9 & $-20.44(0.088)$ & $-20.20(0.07)^{*}$ \\
\hline Dib_mix1 & $/ / \mathrm{b}$ & 1250 & 66.1 & $-20.30(0.084)$ & $-19.95(0.074)^{*}$ \\
\hline
\end{tabular}

Table 2: Summary of diffusion data for Lu and Hf in diopside. Hafnium data are from Bloch and Ganguly (2014). *Indicates that the diffusion profile was better fit using Eq. (2) than Eq. (1). 
Figure 1:
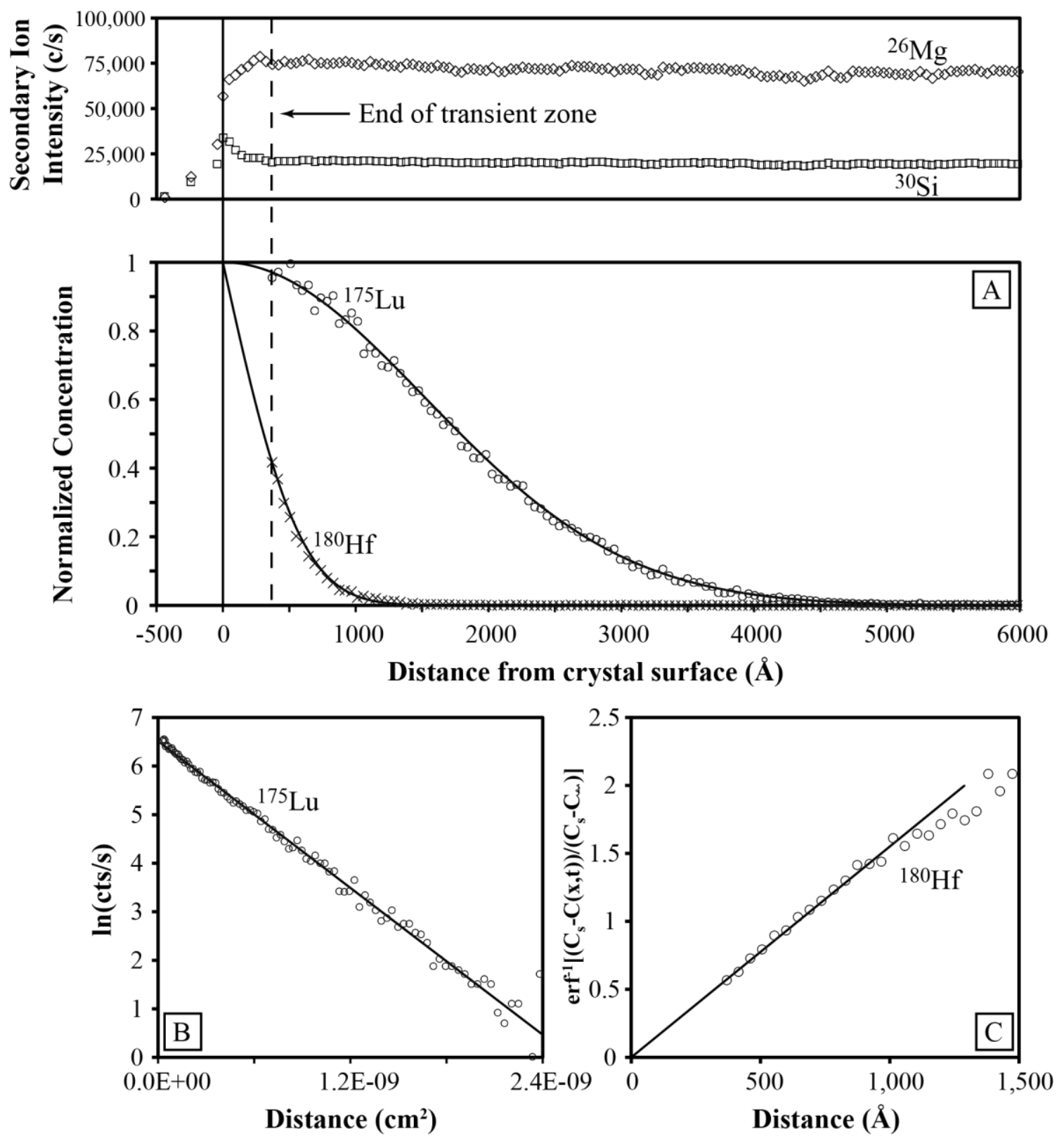
Figure 1: A typical diffusion profile of Lu and Hf and model fits according to Eqs. (1) and (2) in the main text. This diopside was cut perpendicular to the c-crystallographic axis and annealed at $1200{ }^{\circ} \mathrm{C}$ for 91.65 hours. A: Normalized concentration vs. distance plots. B: $\ln (\mathrm{cts} / \mathrm{s})$ vs. distance ${ }^{2}$ plot for Lu, used to linearize model fits obtained using Eq. (2). The diffusion coefficients are extracted from the relation $\alpha=-1 /(4 \mathrm{Dt})$, where $\alpha=$ slope. C: Plot of $\operatorname{erf}^{-1}\left[\left(\mathrm{C}_{\mathrm{S}}-\mathrm{C}(\mathrm{x}, \mathrm{t})\right) /\left(\mathrm{C}_{\mathrm{S}}-\mathrm{C}_{\infty}\right)\right]$, used to linearize model fits to the diffusion data obtained using Eq. (1). Diffusion coefficients are extracted from the linearized data according to $\alpha=(4 \mathrm{Dt})^{-1 / 2}$. 
Figure 2:

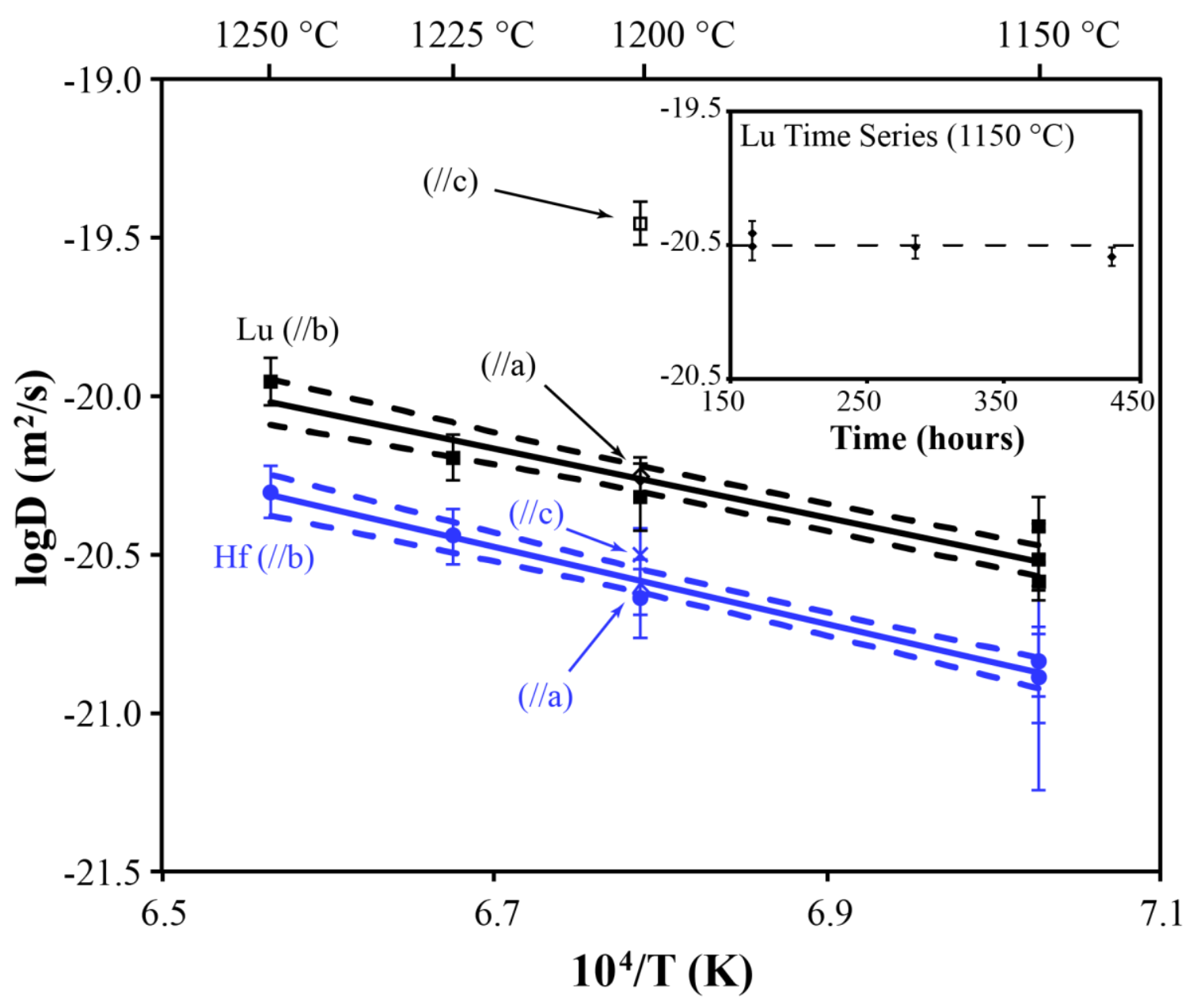

Figure 2: Retrieved diffusion coefficients and modeled Arrhenius trends. Inset shows Lu time series experiments run at $1150{ }^{\circ} \mathrm{C}$. Hafnium data are from Bloch and Ganguly (2014). 
Figure 3:

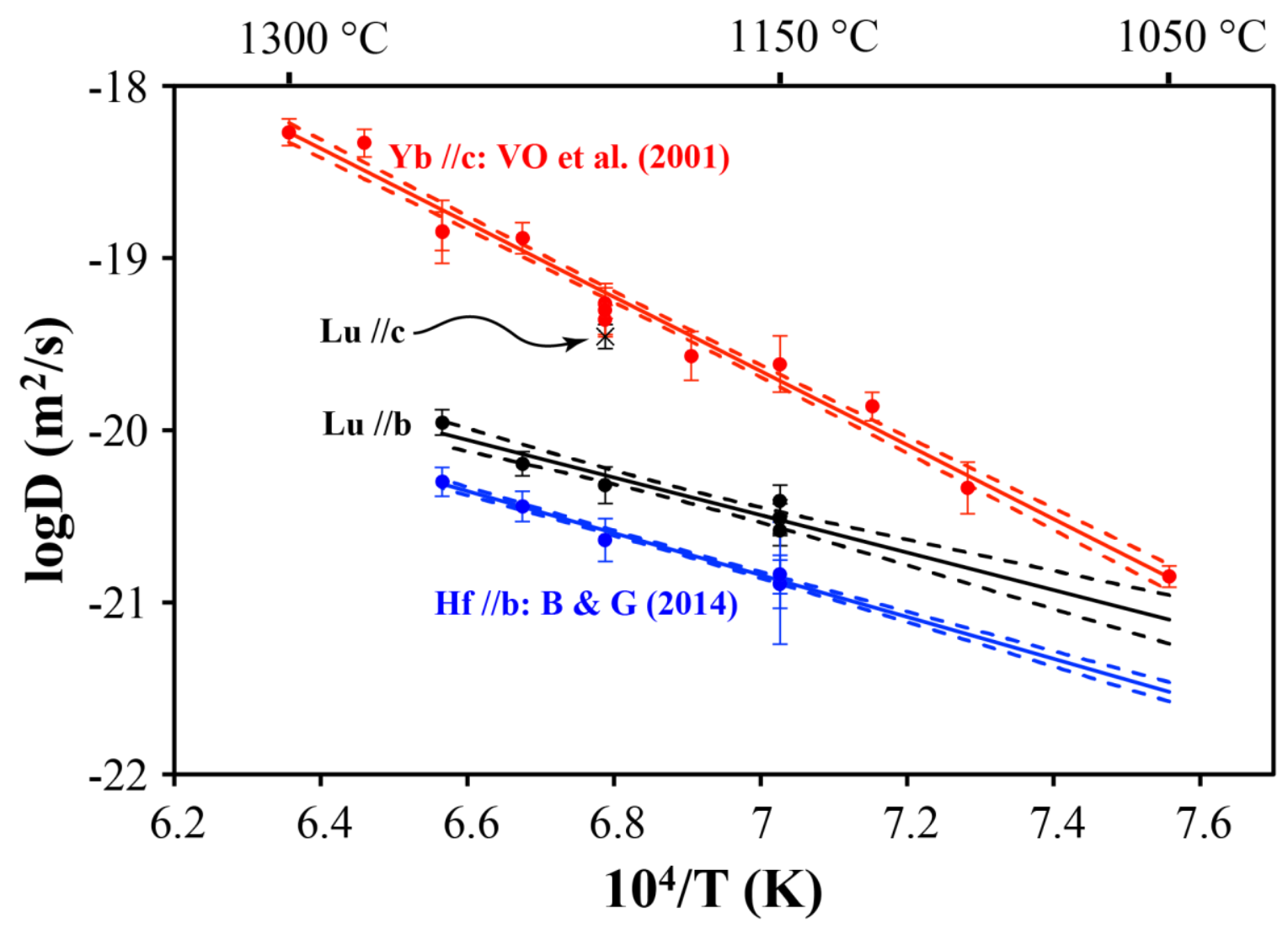

Figure 3: Lutetium and hafnium data collected in this study and data for $\mathrm{Yb}$ diffusion parallel to the c-crystallographic axis in diopside reported by Van Orman et al. (2001). Dashed lines represent the $2 \sigma$ error envelopes ( $~ 95 \%$ confidence intervals). Lutetium and hafnium data have been extrapolated from the range of experimental temperatures (1150$1250{ }^{\circ} \mathrm{C}$ ) down to $1050^{\circ} \mathrm{C}$. 
Figure 4:
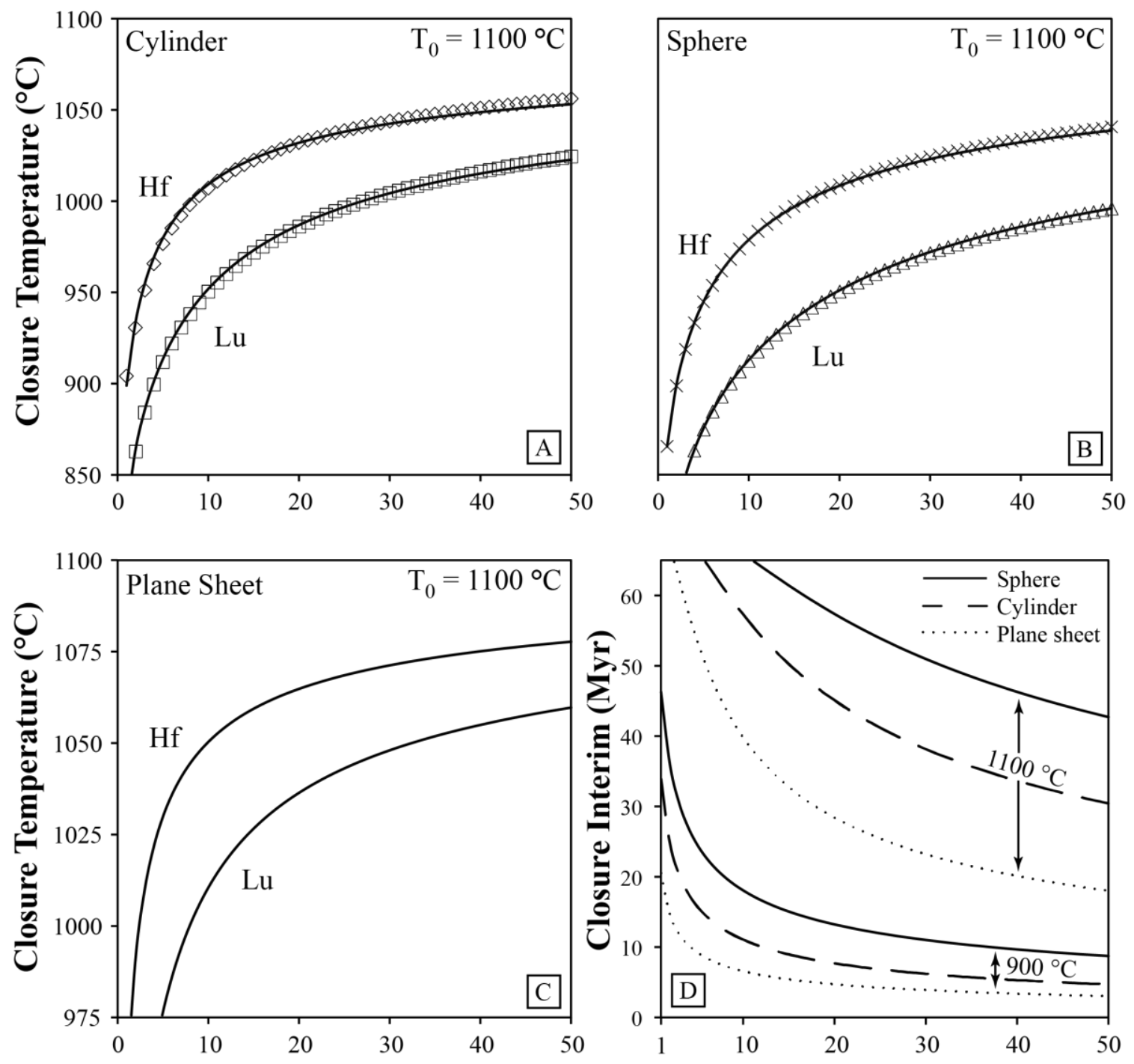

Initial Cooling Rate $\left({ }^{\circ} \mathrm{C} / \mathrm{Myr}\right)$ 
Figure 4: Closure temperatures and resulting closure interims for $\mathrm{Lu}$ and $\mathrm{Hf}$ in diopside. Closure temperatures for Lu and $\mathrm{Hf}$ in cylindrical, spherical and plane sheet geometries are illustrated in A, B and C, respectively. Curves in Figs. A-C are all calculated for clinopyroxene with a peak temperature of $1100{ }^{\circ} \mathrm{C}$ and a characteristic grain size (radius or half-width) of $1 \mathrm{~mm}$. Markers in A and B represent numerical calculations of Lu and Hf closure temperatures using the model of Bloch and Ganguly (2015). D: Closure interims (see text for definition) for $1 \mathrm{~mm}$ pyroxene. Peak temperatures and geometries for each curve are labeled as such. 
Figure 5:

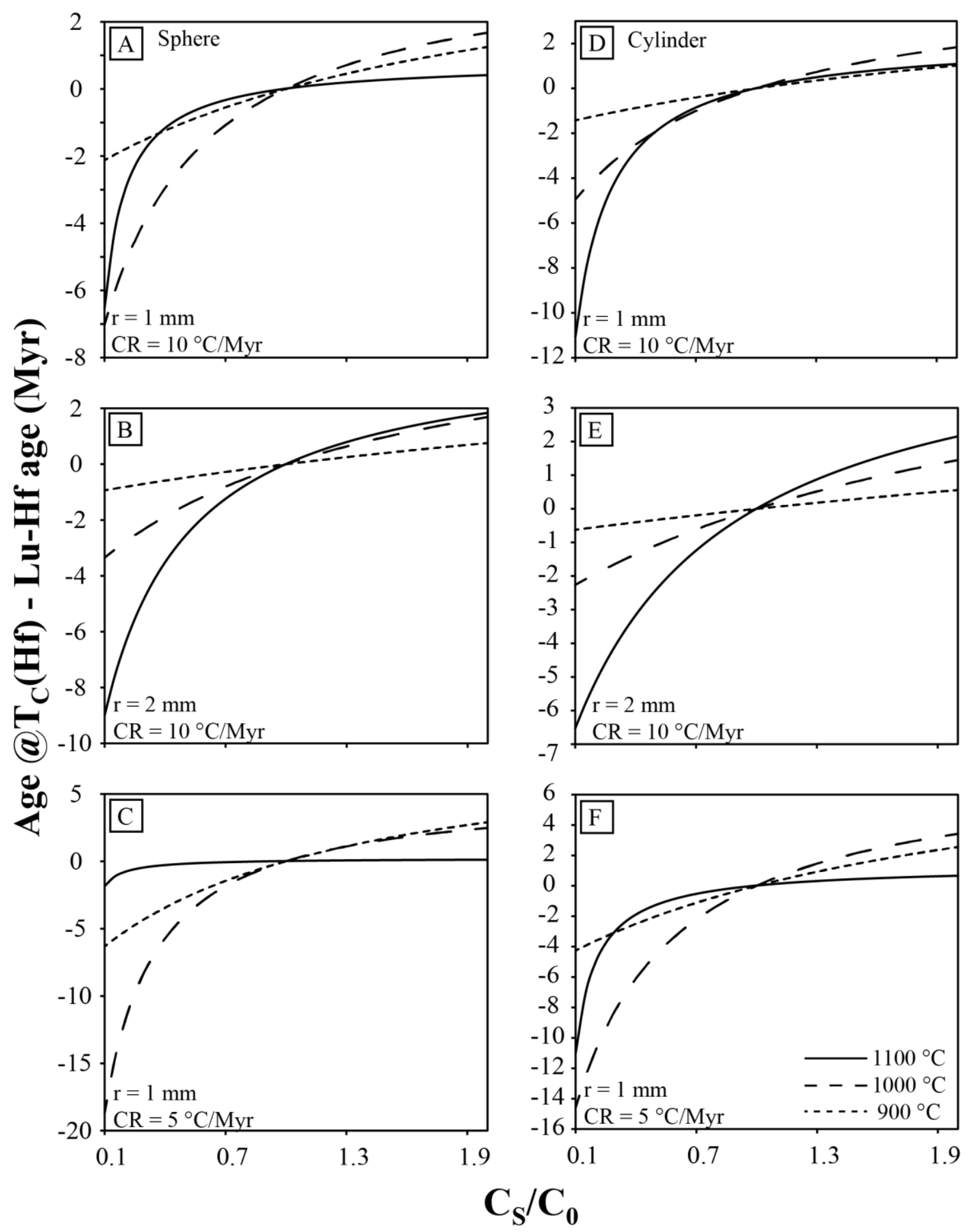

Figure 5: Deviation of the recorded Lu-Hf age from the age of Hf closure in clinopyroxene for spherical (A-C) and Cylindrical (D-F) geometries. The imposed peak 
temperature, grain size and cooling rate labeled in each panel. CR denotes cooling rate and $\mathrm{r}$ denotes radius. $\mathrm{C}_{0}=$ homogenous $\mathrm{Lu}$ concentration at $\mathrm{t}=0, \mathrm{Cs}=$ fixed $\mathrm{Lu}$ surface concentration at $\mathrm{t}>0$. 
Figure 6:

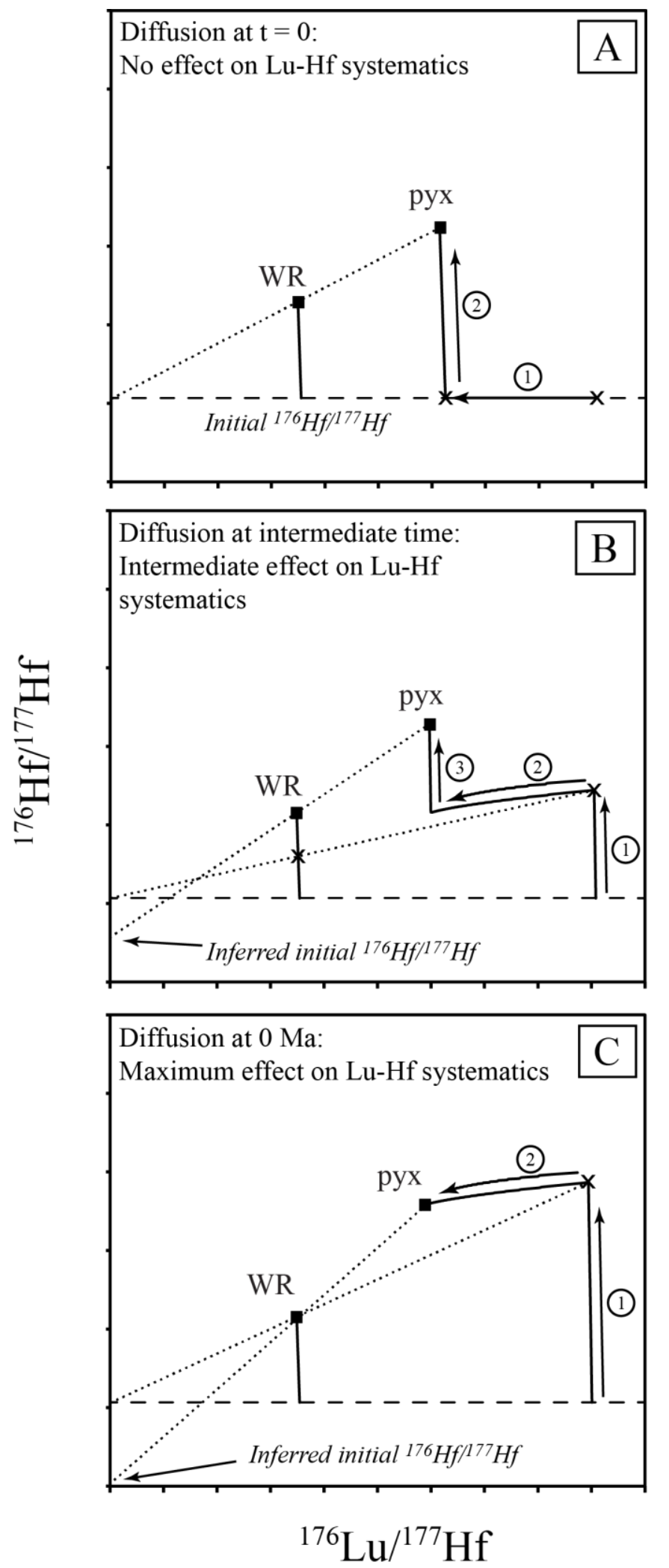


Figure 6: Schematic illustration of the importance of the timing of reheating episodes on the Lu-Hf age recorded in clinopyroxene. A: Lower end-member. Diffusion occurs instantaneously at the instant radiogenic ${ }^{176} \mathrm{Hf}$ begins to accumulate (1), and there is no impact on the recorded Lu-Hf age as all ${ }^{176} \mathrm{Hf}$ ingrowth occurs after Lu loss (2). B: Intermediate (realistic) scenario, where reheating occurs sometime after accumulation of radiogenic ${ }^{176} \mathrm{Hf}(1=$ initial closed-system decay/ingrowth; $2=\mathrm{Lu}$ loss via during reheating; 3 = further closed-system decay/ingrowth after reheating). C: Upper endmember. All closed system decay/ingrowth (1) occurs after reheating event (2), which occurs instantaneously at $0 \mathrm{Ma}$. This hypothetical scenario would result in the maximum disturbance of the recorded Lu-Hf age. 
Figure 7:
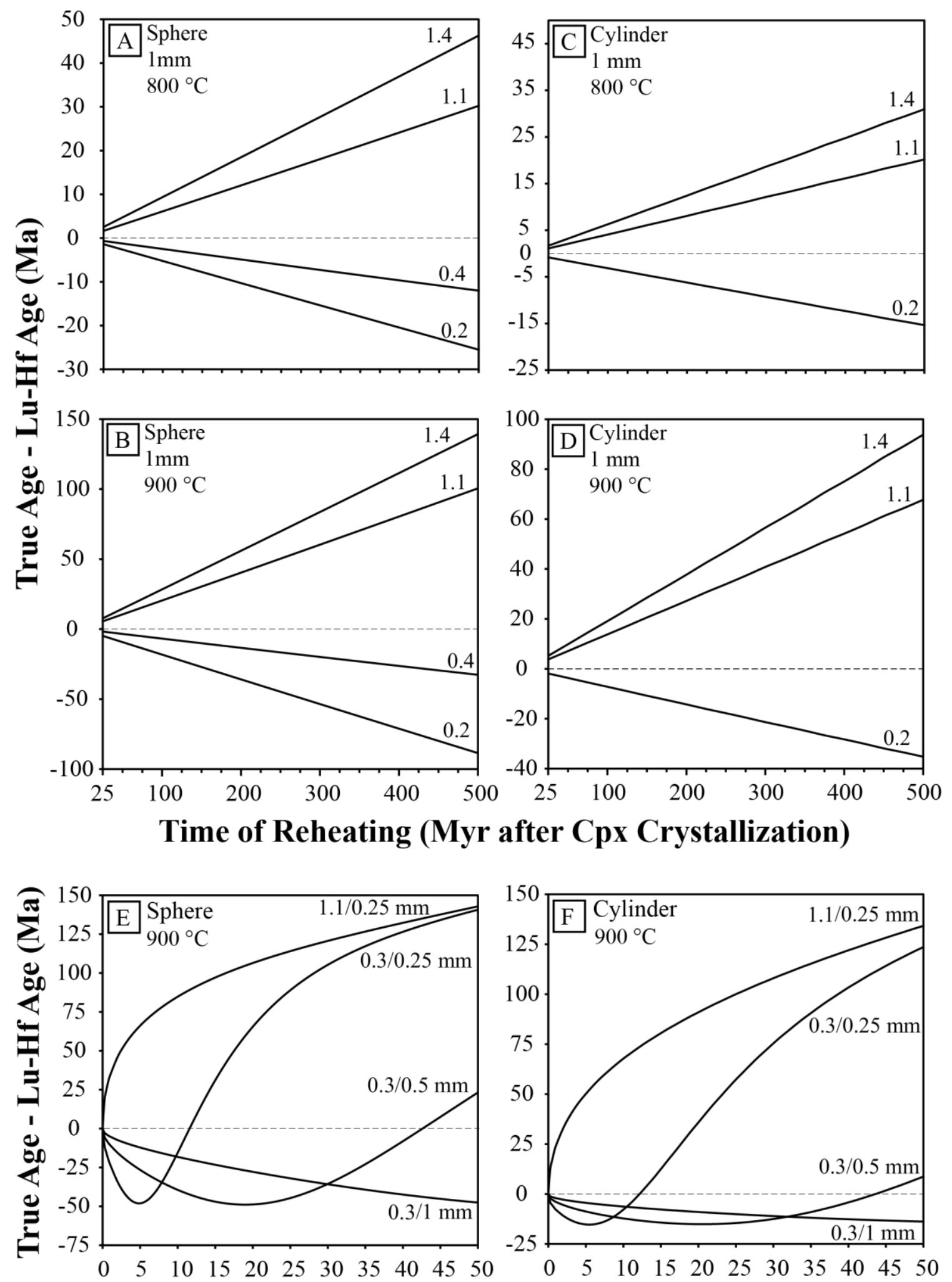

Duration of Reheating (Myr) 
Figure 7: Effect of the timing and duration of reheating episodes on Lu-Hf systematics, plotted in terms of true age - Lu-Hf age (see main text for further explanation). A-B and C-D show the effect of delaying the onset of the reheating episode in spherical and cylindrical geometries, respectively. All simulations illustrated in A-D impose a reheating duration of 5 Myr. The grain size (radius) and temperature of reheating are noted in each panel, and the numbers on each curve represent $\mathrm{C}_{\mathrm{S}} / \mathrm{C}_{0}$. Panels $\mathrm{E}$ and $\mathrm{F}$ show the effects of varying the duration of reheating in spherical and cylindrical geometries, respectively. All simulations in $\mathrm{E}$ and $\mathrm{F}$ imposed a reheating temperature of $900{ }^{\circ} \mathrm{C}$ beginning $100 \mathrm{Myr}$ after cpx crystallization, and the numbers on each curve represent $\mathrm{C}_{\mathrm{S}} / \mathrm{C}_{0} /$ radius. 
Figure 8:

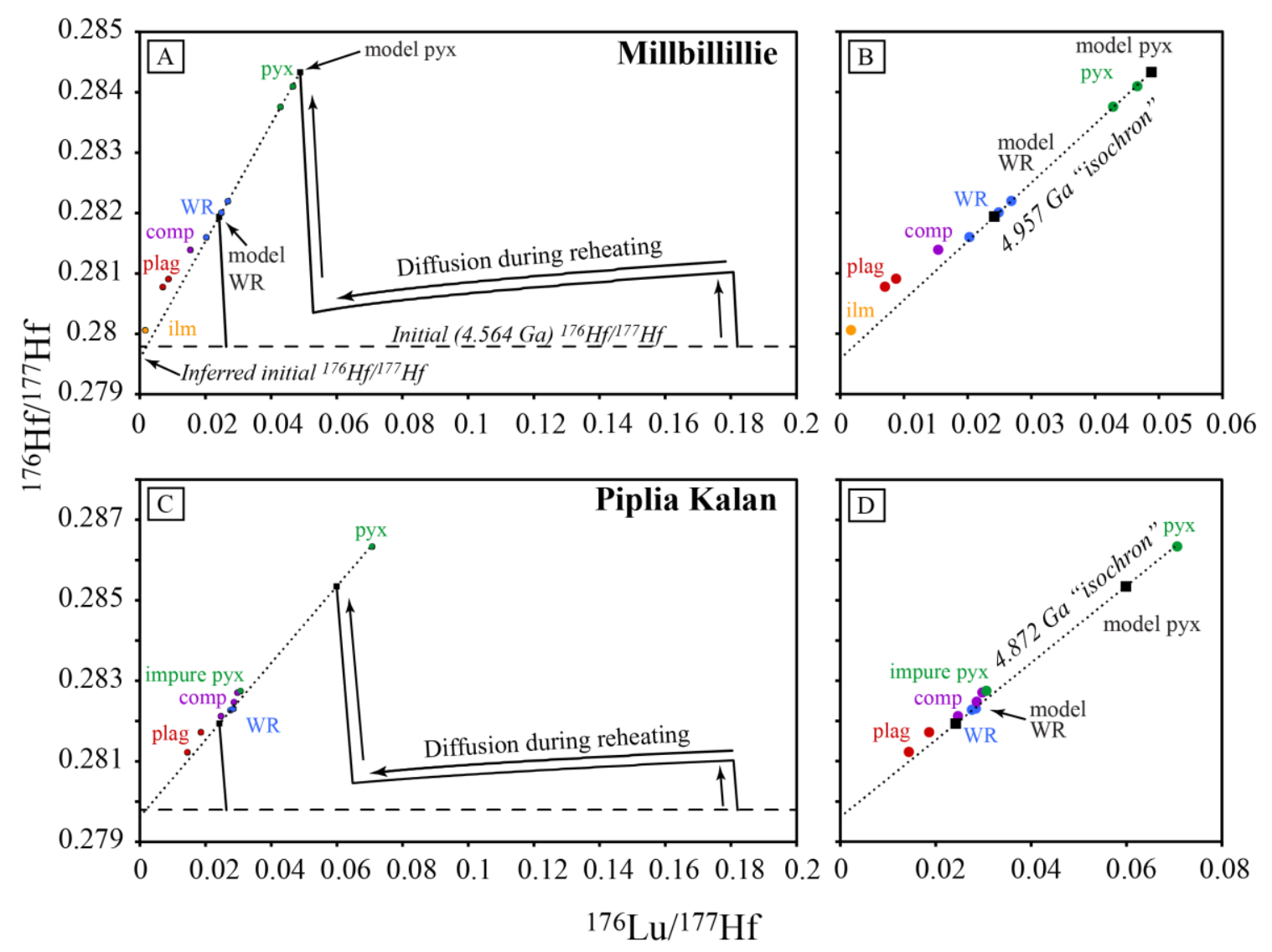

Figure 8: Model simulations for the development of pyroxene-bulk-rock Lu-Hf isochrons of Millbillillie and Piplia Kalan. A: Millbillillie. Solid lines show the modeled ${ }^{176} \mathrm{Hf} /{ }^{177} \mathrm{Hf}$ vs. ${ }^{176} \mathrm{Lu} /{ }^{177} \mathrm{Hf}$ evolution of whole rock and pyroxene. Arrows indicate periods of closed system evolution (unlabeled) or diffusive exchange. Composite (comp), ilmenite (ilm), plagioclase (plag), pyroxene (pyx) and whole-rock (WR) data are from Bast et al. (2012). B: Enlarged plot showing the data reported by Bast et al. (2012) and the numerically calculated isochron. C and D: Model simulations for the eucrite Piplia Kalan. Arrows and data abbreviations are the same as in A and B. 
Figure 9:

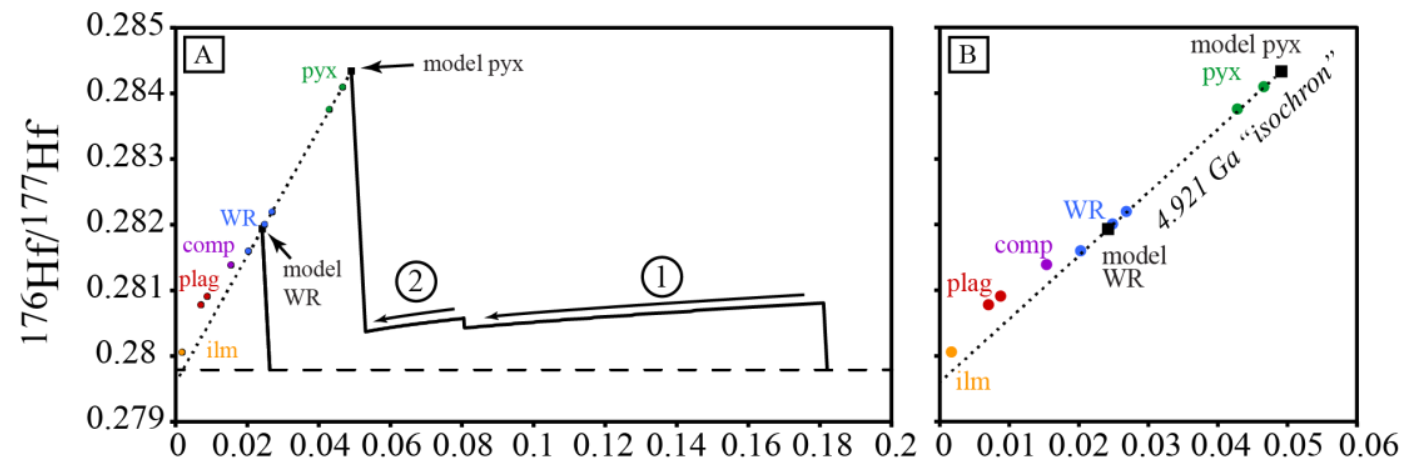

${ }^{176} \mathrm{Lu} /{ }^{177} \mathrm{Hf}$
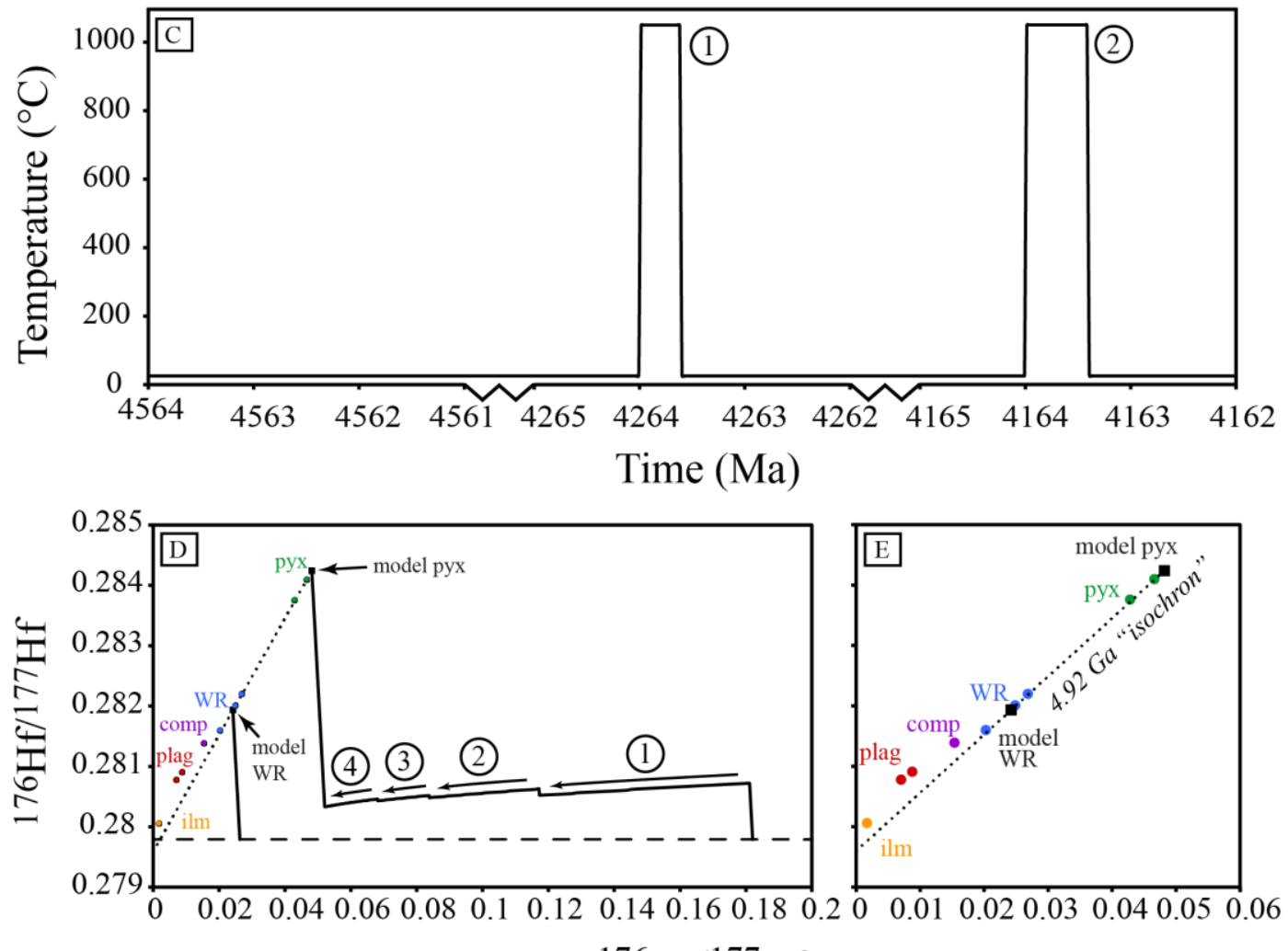

${ }^{176} \mathrm{Lu} /{ }^{177} \mathrm{Hf}$

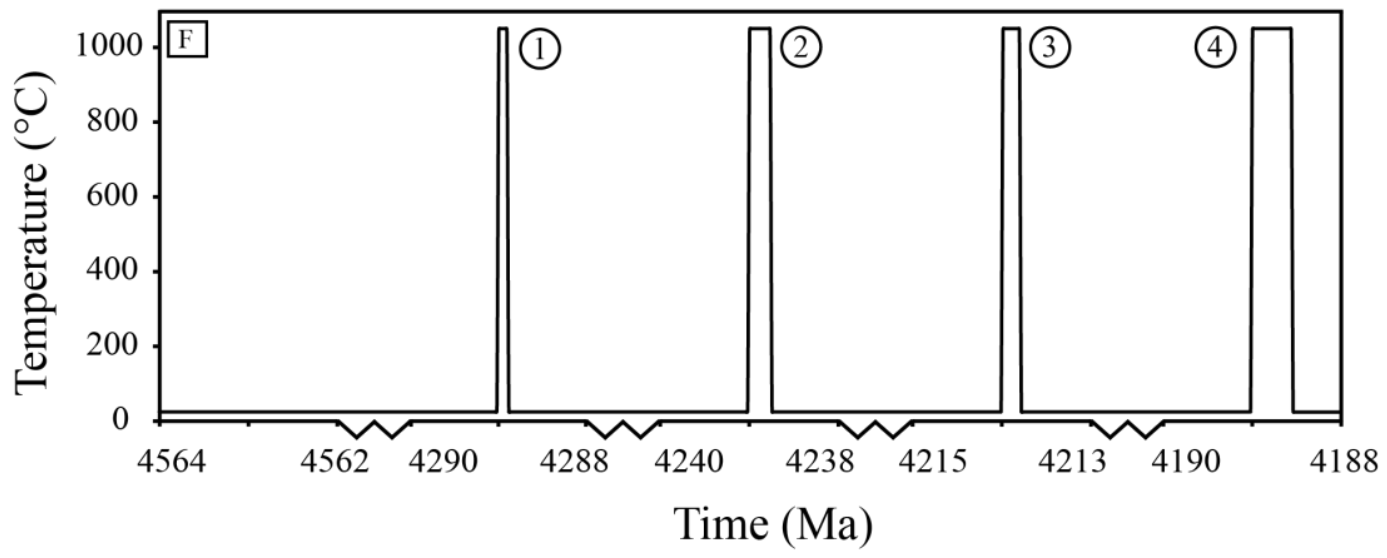


Figure 9: Effect of multiple shorter thermal pulses on the modeled Lu-Hf ages of Millbillillie. Data are again from Bast et al. (2012). A: evolution of bulk ${ }^{176} \mathrm{Hf} /{ }^{177} \mathrm{Hf}$ vs. ${ }^{176} \mathrm{Lu} /{ }^{177} \mathrm{Hf}$ in pyroxene through two thermal pulses, represented by the temperature-time path illustrated in $\mathrm{C}$. The number on each arrow in A indicates diffusive mobilization that occurs during the correspondingly numbered thermal pulse in $\mathrm{C}$. The resulting isochron, enlarged in panel $\mathrm{B}$, does not differ substantially from the isochron calculated assuming a single thermal pulse (Figs. 8A-B). Panels D-F are the same as A-C; only four thermal pulses are imposed instead of two. 
Figure 10:

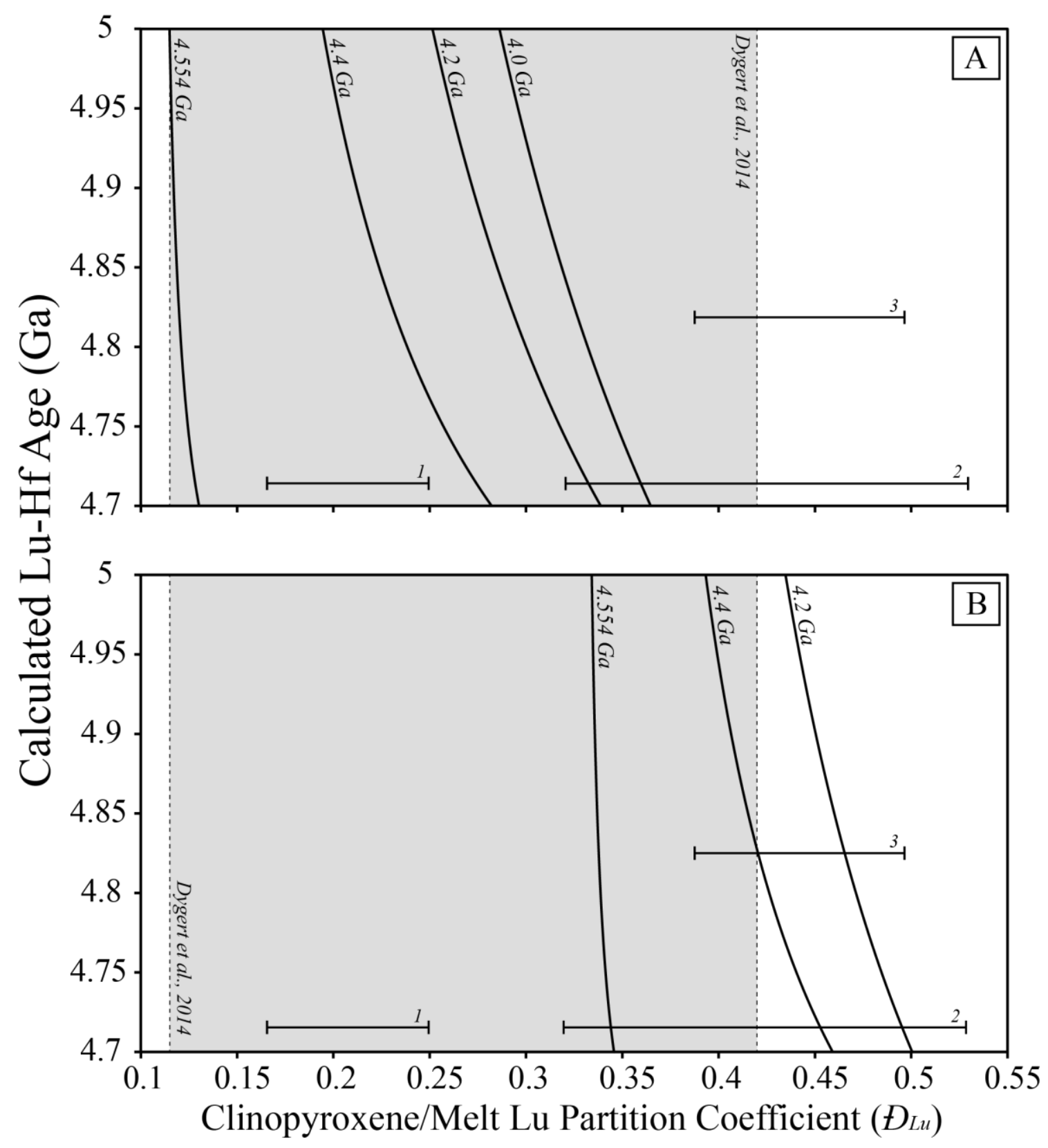


Figure 10: Model pyroxene-whole-rock Lu-Hf ages for Millbillillie as a function of the timing of metamorphism and the Lu partition coefficient $\left(\bigoplus_{L u}\right)$ between clinopyroxene and the surrounding melt phase. Each curve represents simulations imposing a specific time at which metamorphism occurred, and is labeled as such. Shaded area represents the range of experimentally determined $\bigoplus_{L u}$ values reported by Dygert et al. (2014).

Horizontal bars show the range of experimentally determined $\bigoplus_{Y b}$ values reported by ${ }^{l}$ Blundy and Dalton (2000), ${ }^{2}$ Johnston and Schwab (2004) and ${ }^{3}$ Laubier et al. (2014). A and B show calculations imposing initial ${ }^{176} \mathrm{Lu} /{ }^{177} \mathrm{Hf}$ ratios in pyroxene of 0.18 and 0.086 , respectively (see text for further explanation). 
Figure 11:

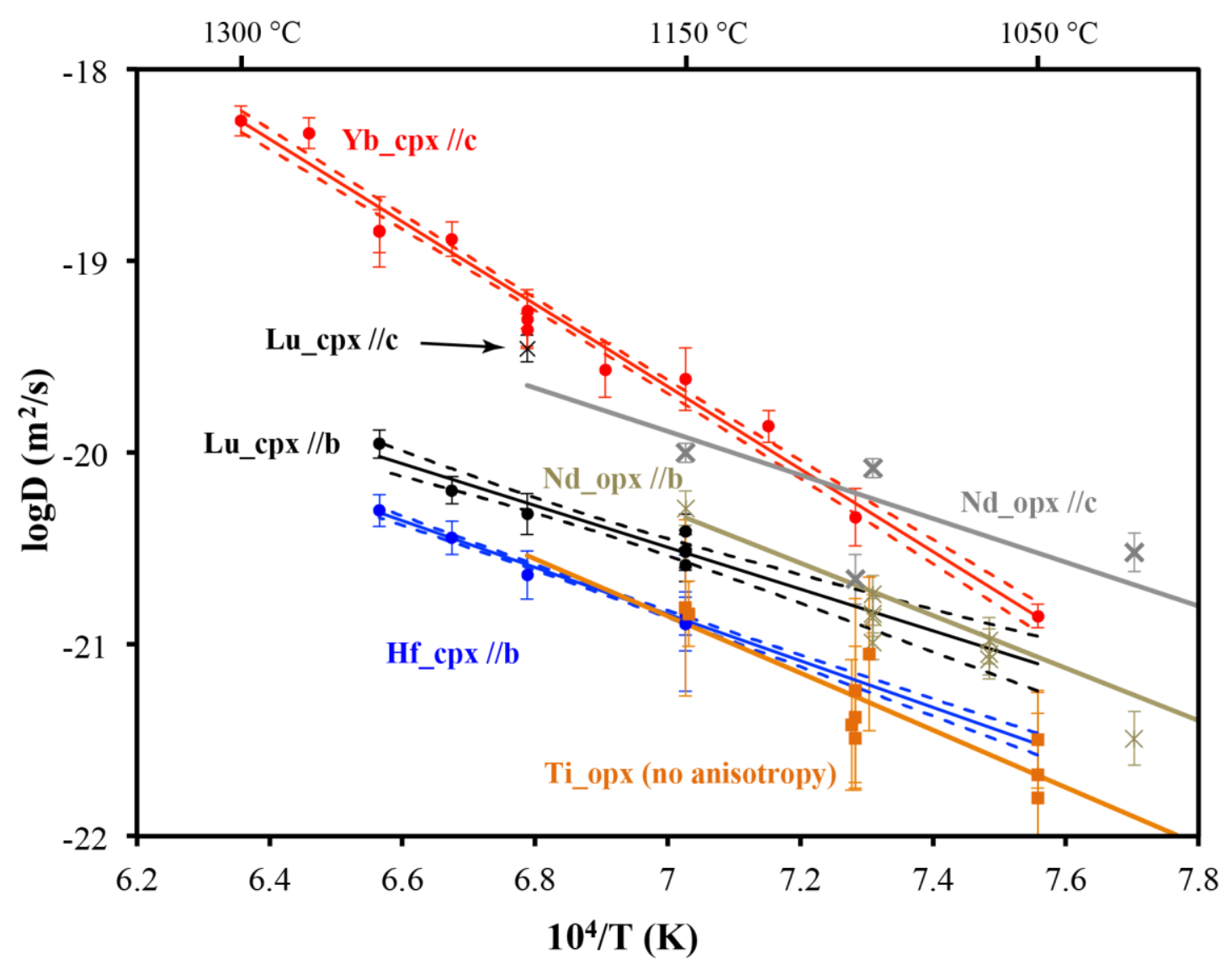

Figure 11: Comparison between REE, Hf and Ti diffusion in diopside and enstatite. Lutetium and hafnium data are from this study; $\mathrm{Yb}, \mathrm{Nd}$ and $\mathrm{Ti}$ data are from Van Orman et al. (2001), Sano et al. (2011) and Cherniak and Liang (2012), respectively. Dashed lines represent the $2 \sigma$ error envelopes ( $\sim 95 \%$ confidence intervals), as in Figs 2 and 3. 